\title{
Principles of Intrinsic Motor Cortex Connectivity in Primates
}

\author{
Nicholas S. Card ${ }^{1,3,4}$ and Omar A. Gharbawie ${ }^{1,2,3,4}$ \\ ${ }^{1}$ Systems Neuroscience Center, ${ }^{2}$ Department of Neurobiology, University of Pittsburgh, Pittsburgh, 15213, PA, ${ }^{3}$ Department of Bioengineering, \\ University of Pittsburgh, Pittsburgh, 15260, PA, and ${ }^{4}$ Center for Neural Basis of Cognition, University of Pittsburgh, Pittsburgh, 15213, PA
}

The forelimb representation in motor cortex (M1) is an important model system in contemporary neuroscience. Efforts to understand the organization of the M1 forelimb representation in monkeys have focused on inputs and outputs. In contrast, intrinsic M1 connections remain mostly unexplored, which is surprising given that intra-areal connections universally outnumber extrinsic connections. To address this knowledge gap, we first mapped the M1 forelimb representation with intracortical microstimulation (ICMS) in male squirrel monkeys. Next, we determined the connectivity of individual M1 sites with ICMS + intrinsic signal optical imaging (ISOI). Every stimulation site activated a distinctive pattern of patches $(\sim 0.25$ to $1.0 \mathrm{~mm}$ radius) that we quantified in relation to the motor map. Arm sites activated patches that were mostly in arm zones. Hand sites followed the same principle, but to a lesser extent. The results collectively indicate that preferential connectivity between functionally matched patches is a prominent organizational principle in M1. Connectivity patterns for a given site were conserved across a range of current amplitudes, train durations, pulse frequencies, and microelectrode depths. In addition, we found close correspondence in somatosensory cortex between connectivity that we revealed with ICMS+ISOI and connections known from tracers. ICMS+ISOI is therefore an effective tool for mapping cortical connectivity and is particularly advantageous for sampling large numbers of sites. This feature was instrumental in revealing the spatial specificity of intrinsic M1 connections, which appear to be woven into the somatotopic organization of the forelimb representation. Such a framework invokes the modular organization well-established for sensory cortical areas.

Key words: effective connectivity; intrinsic signal optical imaging; microstimulation; motor cortex; motor map; somatosensory cortex

Significance Statement

Intrinsic connections are fundamental to the operations of any cortical area. Surprisingly little is known about the organization of intrinsic connections in motor cortex (M1). We addressed this knowledge gap using intracortical microstimulation (ICMS) concurrently with intrinsic signal optical imaging (ISOI). Quantifying the activation patterns from dozens of M1 sites allowed us to uncover a fundamental principle of M1 organization: M1 patches are preferentially connected with functionally matched patches. Relationship between intrinsic connections and neurophysiological map is well-established for sensory cortical areas, but our study is the first to extend this framework to M1. Microstimulation+imaging opened a unique possibility for investigating the connectivity of dozens of tightly spaced M1 sites, which was the linchpin for uncovering organizational principles.

Received Dec. 31, 2019; revised Apr. 2, 2020; accepted Apr. 6, 2020.

Author contributions: N.S.C. and 0.A.G. designed research; N.S.C. and 0.A.G. performed research; N.S.C. and 0.A.G. analyzed data; N.S.C. and O.A.G. wrote the paper.

This work was supported by funds from the University of Pittsburgh Brain Institute, National Institutes of Health Grants R00 NS079471 and R01 NS105697, the Whitehall Foundation (2017-12-94). We thank ToniAnn Zullo for outstanding animal care before, after, and during all procedures; Anthony Sloan for support during initial experiments; and Dr. Peter L. Strick for many thoughtful discussions related to this study.

The authors declare no competing financial interests.

Correspondence should be addressed to Omar A. Gharbawie at omar@pitt.edu.

https://doi.org/10.1523/JNEUROSCI.0003-20.2020

Copyright $\odot 2020$ the authors

\section{Introduction}

Primary motor cortex (M1) is central to arm and hand control in primates. The forelimb representation in monkeys is a widelyused model for studying cortical control of movement, neural basis of learning, population coding, and more. To understand the spatial organization of M1 networks, neuroanatomical and neurophysiological investigations have focused on the inputs and outputs of the M1 forelimb representation (Stepniewska et al., 1993; He et al., 1995; Park et al., 2001; Dum and Strick, 2005). Such efforts have been instrumental in shaping our understanding of how the M1 forelimb representation is connected with the rest of the brain and with the spinal cord. In contrast, far less is 
Table 1. Procedures and data collected from each animal

\begin{tabular}{|c|c|c|c|c|c|c|}
\hline Monkey & Hemisphere & Procedures & Imaging runs & Effective connectivity sites & Motor map sites & $\overline{\text { Receptive field sites }}$ \\
\hline M & Left & 14 & 58 & 49 & 223 & 87 \\
\hline \multirow[t]{2}{*}{ D } & Left & 4 & 11 & 11 & 108 & 1 \\
\hline & Right & 3 & 15 & 1 & 23 & 17 \\
\hline $\mathrm{R}$ & Left & 6 & 20 & 18 & 131 & - \\
\hline G & Left & 2 & 2 & 2 & - & 35 \\
\hline Total & & 29 & 106 & 81 & 485 & 140 \\
\hline
\end{tabular}

Numbers for each animal are limited to the data included in the present study.

known about the organization of the intrinsic connections of the M1 forelimb representation. Mapping these connections and the networks that they establish would provide a useful framework for interrogating the computations that occur within M1 in the service of arm and hand control.

Only a few studies have examined the organization of the intrinsic networks of the M1 forelimb representation (Huntley and Jones, 1991; Keller, 1993; Capaday et al., 1998, 2009). Huntley and Jones (1991) showed in macaque monkeys that patchy horizontal connections link the M1 digit zone with other hand and arm zones. A similar connectivity pattern was reported in cats for the M1 wrist zone (Keller, 1993). Both studies therefore provided evidence that M1 horizontal connections are spatially selective (i.e., patchy) and at the same time distributed throughout the forelimb representation. However, neither study quantified the traced connections, which complicates our understanding of the relationship between intrinsic M1 connections and somatotopy of the forelimb representation. Even if the results were quantified, the findings may have only shed light on the connectivity of hand zones (i.e., digit and wrist) given that connections of the M1 forelimb representation are likely zone specific (Dea et al., 2016; Hamadjida et al., 2016). The density of intrinsic connections in sensory cortical areas and their role in functional binding (Douglas and Martin, 2004) leave little doubt that our limited understanding of intrinsic M1 connectivity represents a critical knowledge gap about M1 functional architecture.

The present study was motivated by a need for understanding the principles that govern the organization of intrinsic M1 networks. Our main objectives were to (1) determine connectivity for sites throughout the M1 forelimb representation, and (2) quantify the relationship between connectivity and somatotopy of the M1 forelimb representation. To accomplish these objectives, we adopted an imaging-based approach in squirrel monkeys that allowed us to investigate $\mathrm{M} 1$ connectivity in vivo (Stepniewska et al., 2011; Brock et al., 2013). First, we mapped the M1 forelimb representation using intracortical microstimulation (ICMS). Next, we stimulated M1 sites individually and measured the evoked cortical response using intrinsic signal optical imaging (ISOI). Finally, we quantified the activation maps in relation to somatotopy of the $\mathrm{M} 1$ forelimb representation as a measure of intrinsic M1 connectivity. Our observations from dozens of stimulation sites showed that intrinsic M1 connectivity is mostly patchy and the patches are spatially organized with respect to the forelimb motor map.

\section{Materials and Methods}

\section{Animals}

Experiments were performed on four adult male squirrel monkeys (Saimiri sciureus). Animals were four to eight years old and weighed between 800 and $1200 \mathrm{~g}$. All procedures were approved by the University of Pittsburgh Institutional Animal Care and Use Committee and followed the guidelines of the National Institutes of Health guide for the care and use of laboratory animals.

\section{Surgical procedures}

One cranial opening per studied hemisphere provided access to frontal and parietal cortex. Each cranial opening was accessed multiple times under sterile conditions (Table 1 ). Thirty minutes before sedation, animals were treated with an antiemetic (Zofran, $0.3 \mathrm{mg} / \mathrm{kg}$, i.m.) and Atropine $(0.03 \mathrm{mg} / \mathrm{kg}, \mathrm{i} . \mathrm{m}$.) to reduce secretions. Ketamine induction $\left(10-15 \mathrm{mg} / \mathrm{kg}\right.$, i.m.) was followed with isoflurane $(0.5-2.5 \%)$ in $\mathrm{O}_{2}(2-3$ $1 / \mathrm{min})$. Once sedated, dexamethasone (1 mg/kg, i.m.), Ketofen $(2 \mathrm{mg} / \mathrm{kg}$, i.m.), and gentamicin ( $2 \mathrm{mg} / \mathrm{kg}$, i.m.) were administered to prevent brain swelling, pain, and infection, respectively. Animals were intubated, artificially ventilated, wrapped in a heating blanket, and secured in a stereotaxic frame. Heart rate, arterial oxygen saturation, expired $\mathrm{CO}_{2}$, blood glucose, and body temperature were monitored continuously and adjustments to anesthesia, ventilation, and heat were made accordingly. Fluids ( $5 \%$ dextrose in lactated Ringer's solution, $2-3 \mathrm{ml} / \mathrm{kg} / \mathrm{h}$, i.v.) were provided for the duration of each procedure.

An incision was performed for each procedure. All steps from this point onwards were conducted with the aid of a surgical microscope. For the first procedure within a hemisphere, a dental drill was used to open a rectangular window for access to the forelimb representations in motor cortex and in somatosensory cortex. After a durotomy, cortical pulsations were stabilized with $3 \%$ agarose (Invitrogen) solution in physiological saline. Data acquisition proceeded from this point and lasted for several hours. During data acquisition, anesthesia was maintained with ketamine infusion $(3-6 \mathrm{mg} / \mathrm{kg} / \mathrm{h}$, i.v.) and isoflurane $(0.25-1.0 \%)$ mixed in $50 \% \mathrm{~N}_{2} \mathrm{O}_{2}$ and $50 \% \mathrm{O}_{2}(2-3 \mathrm{l} / \mathrm{min})$. At all other times, anesthesia was maintained with isoflurane $(0.5-2.5 \%)$ mixed in $\mathrm{O}_{2}(2-3 \mathrm{l} / \mathrm{min})$. The agar was removed at the conclusion of data acquisition and an artificial dura (Tecoflex; Sakas et al., 1990) was secured into the cranial opening to protect the cortex. The craniotomy was sealed with dental cement and the scalp was sutured closed. The animal was recovered from anesthesia and another dose of the pre-procedure drugs was administered in addition to vitamin B12 (0.5 mg/kg, i.m.). Analgesics and corticosteroids were administered every $12 \mathrm{~h}$ for the following $72 \mathrm{~h}$. Each procedure lasted $\sim 14 \mathrm{~h}$ from sedation to recovery and the single terminal experiment lasted $43 \mathrm{~h}$

\section{Motor mapping}

The objective was to map the forelimb representation in M1 with ICMS. A hydraulic microdrive (Narishige MO-10) connected to a Kopf micromanipulator was used for positioning a platinum/iridium microelectrode (125 $\mu \mathrm{m}$ shaft, $260 \mathrm{k} \Omega$ median impedance, MicroProbes). Electrode impedance was periodically tested in each motor mapping session and a new microelectrode was used if impedance approached $1 \mathrm{M} \Omega$.

Each penetration targeted the approximate depth of layer $\mathrm{V}$ (1800 $\mu \mathrm{m}$ below cortical surface). Microstimulation trains (18 cathodal pulses, $0.2 \mathrm{~ms}$ pulse width, $300 \mathrm{~Hz}$ pulse frequency, $1 \mathrm{~Hz}$ train frequency) were delivered with an 8-Channel Stimulator (model 3800, AM Systems). Current amplitude was adjusted with a stimulus isolation unit (model BSI-2A, BAK Electronics) until a movement was evoked and up to a maximum of $150 \mu \mathrm{A}$. The threshold recorded for each site was the minimum current amplitude that evoked movement on $\sim 50 \%$ of microstimulation trains. Responses to ICMS were generally suppressed with heart rate $<250$ beats/min. The combination of ketamine infusion and 

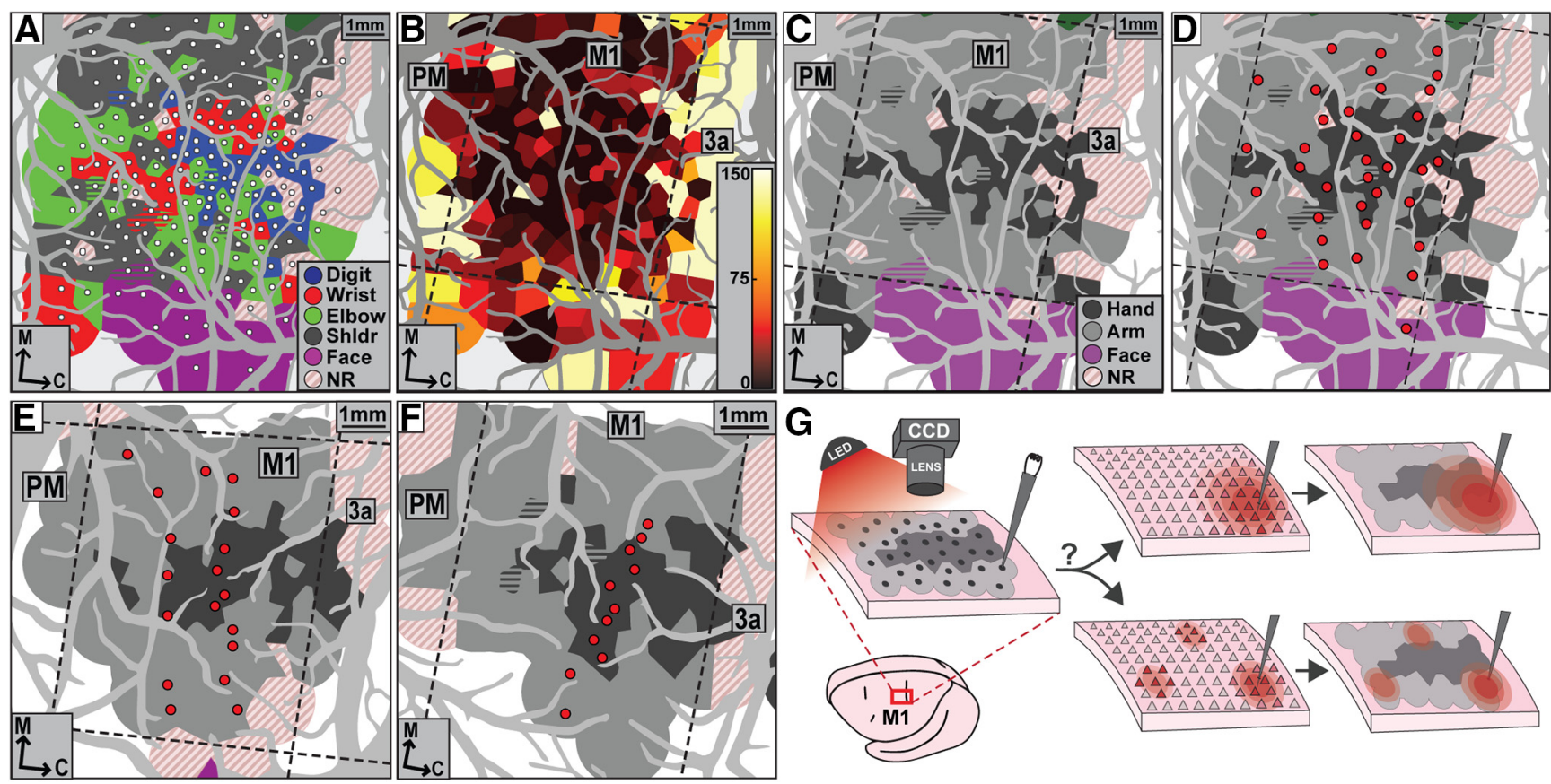

Figure 1. Mapping M1 connectivity with microstimulation and imaging. $\boldsymbol{A}$, Map of the M1 forelimb representation in the left hemisphere (monkey M). Major blood vessels are masked in gray. White dots depict microelectrode sites ( $n=223$ ) for ICMS. Motor map was completed over the course of multiple experiments (30-60 ICMS sites/experiment). Voronoi tiles (1.0 mm radius) are color-coded according to the ICMS-evoked movement. Striped tiles (1 color/joint) represent dual movements. Non-responsive sites "NR" failed to evoke movements with current amplitudes up to $150 \mu \mathrm{A}$. B , Same motor map in $\boldsymbol{A}$, but color-coded according to current amplitude ( $\mu \mathrm{A})$ for evoking movements. Rostral and caudal M1 borders (dotted lines) are drawn at the transition from low $(\sim 50 \mu \mathrm{A})$ to high $(\sim 80 \mu \mathrm{A})$ current thresholds. C, Same motor map as in $\boldsymbol{A}$, but wrist and digit sites are now classified as Hand (dark gray); shoulder and elbow sites are now classified as Arm (light gray). $\boldsymbol{D}$, Connectivity mapping sites (red dots) superimposed onto the motor map. $\boldsymbol{E}, \boldsymbol{F}$, Maps of the M1 forelimb representations from monkey $R$ (131 sites) and monkey D (108 sites), respectively. Red dots are connectivity mapping sites. G, Schematic for mapping the connectivity of an M1 site using ICMS+ISOI. Bifurcated arrow points to two potential activation patterns. Top, Activation (red shading) is most intense near the stimulation site and declines with distance. Bottom, Activation is in multiple patches. Right most column, Activation maps co-registered with the motor map for quantification.

isoflurane was central to achieving a state in which ICMS could reliably evoke movements under anesthesia.

ICMS facilitates and suppresses activity in groups of muscles that lead to observable movements around one or more joints. Two to three experimenters evaluated the evoked responses; only one experimenter was not blind to the microelectrode location. Each ICMS site was classified according to the joint (digit, wrist, elbow, shoulder) and movement type (e.g., flexion, extension, abduction, etc.) that showed the most robust effect at the lowest current amplitude. Although shoulder responses were readily detected, the type of shoulder movement evoked was often complicated due to positioning within the stereotaxic apparatus. Forearm responses (i.e., pronation and supination) were included with elbow.

The forelimb representation was mapped over the course of several experiments (30-60 ICMS sites/experiment). Distance between ICMS sites was typically $\leq 1 \mathrm{~mm}$ (Fig. $1 \mathrm{~A}$ ). Each site was recorded on a printed, high-resolution photograph of cortex. Some sites were tested in multiple procedures to confirm stability over weeks/months. Color coded maps were generated in MATLAB using a Voronoi diagram (voronoi function) constrained to a $1.0 \mathrm{~mm}$ radius around each site. Medial and lateral borders were drawn to separate the forelimb representation from the trunk and face representations. The rostral M1 border was estimated from current threshold to evoke movements $(\geq 80 \mu \mathrm{A})$ and distance from central sulcus (Fig. 1B). The caudal M1 border was estimated from thresholds to evoke movements and from the location of area $3 \mathrm{a}$, which was mapped as described in the following section. Animals in the present study had a well-defined forelimb representation. In addition, the relative location of the arm zone (i.e., shoulder sites and elbow sites) and the hand zone (i.e., digit sites and wrist sites) was consistent across animals.

\section{Somatosensory mapping}

The objective was to map the forelimb representation in somatosensory cortex with multiunit recordings. For each mapping site, a tungsten microelectrode (125 $\mu \mathrm{m}$ shaft, $500 \mathrm{k} \Omega$ median impedance) was lowered to the approximate depth of Layer IV (700-800 $\mu \mathrm{m}$ below cortical surface). Multiunit activity was amplified $(10,000 \times)$ and filtered (bandpass $300-5000 \mathrm{~Hz}$ ) using an AC Amplifier (Model 2800, AM Systems). The signal was passed through a 50/60 Hz noise eliminator (HumBug, Quest Scientific Instruments Inc.) then visualized on an oscilloscope and broadcasted over a loud speaker. The receptive field for each microelectrode site was determined from modulations in neural activity in response to systematic stimulation of the contralateral forelimb. Receptive fields were classified according to location on the arm or the hand and whether responses were cutaneous (skin contact) or proprioceptive (joint manipulation). Some sites were remapped in multiple procedures to confirm receptive field stability. Somatosensory maps were generated using the same Voronoi function that was used for the motor map.

Cortical borders were estimated from transitions in receptive field properties along the rostro-caudal dimension. Units in areas $3 \mathrm{~b}$ and 1 responded robustly to cutaneous stimulation and had relatively small receptive fields (e.g., single digit phalanx). In contrast, units in area $3 \mathrm{a}$ responded weakly to the manipulation of multiple joints (e.g., entire digit or multiple digits). Area 2 contained a mixture of units that responded to joint manipulation or cutaneous stimulation. The border between areas $3 \mathrm{~b}$ and 1 was estimated from the representations of the pads of the palm that exist in caudal aspects of area $3 \mathrm{~b}$ and rostral aspects of area 1 (Sur et al., 1982).

\section{ISOI}

We used ISOI to measure the ICMS evoked cortical response. Images of cortex were acquired with a camera based on a 12-bit CMOS sensor (Photon Focus). Image acquisition was controlled with an optical imaging system (Imager 3001, Optical Imaging Ltd). Camera frames $(250 \mathrm{~Hz})$ were temporally binned to $5 \mathrm{~Hz}$ except in initial experiments when temporal binning was purposely set to $20 \mathrm{~Hz}$. Frames were not spatially 
binned. We chose a tandem lens combination such that the field-of-view (768 $\times 768$ pixels) would include $\sim 10 \times 10 \mathrm{~mm}$ of cortex $(\sim 13 \mu \mathrm{m} /$ pixel). This field-of-view captured the entire M1 forelimb representation and surrounding cortex at high spatial resolution. A counter-weighted swivel arm was maneuvered to position the camera directly above cortex. Fine angle adjustments were achieved with a three-axis geared head (410 Junior Geared Tripod Head, Manfrotto). Camera position ( $x, y$, and $z$ directions) was translated with independent linear stages. Illumination $(620 \mathrm{~nm})$ for ISOI was provided from three independently controlled LEDs. Image acquisition was periodically paused for evaluating illumination and any necessary adjustments. For spatial reference, blood vessel patterns were imaged (528 $\mathrm{nm}$ illumination) at the start of each imaging run.

\section{Measuring the stimulation evoked response}

To evoke a cortical response from any point in cortex, a microelectrode (platinum/iridium, $125 \mu \mathrm{m}$ shaft) was lowered to $1000 \mu \mathrm{m}$ below cortical surface. Electrode impedance was comparable at the start $($ median $=260 \mathrm{k} \Omega)$ and end of each experiment $($ median $=320 \mathrm{k} \Omega$ ). ICMS and concurrent ISOI were conducted in an event-based design. A given ICMS site was tested on at least 50 blocks. Every block included at least one ICMS condition and one blank condition (i.e., no ICMS). Every condition was presented only once per block (i.e., one trial/condition). Unless otherwise stated, image acquisition lasted for $4 \mathrm{~s} /$ trial with a $12 \mathrm{~s}$ intertrial interval. In ICMS trials, baseline cortical activity was imaged for two data frames (i.e., $400 \mathrm{~ms}$ in $5 \mathrm{~Hz}$ binning; $100 \mathrm{~ms}$ in 20$\mathrm{Hz}$ binning) before stimulation onset. The start of a trial in any condition was synchronized with the ventilation phase to reduce respiration artifact. The ICMS set up was identical to the one used for motor mapping except here we used a voltage-controlled stimulus isolation unit (Model 3820, AM Systems). Unless otherwise stated, the stimulation train in a single ICMS trial consisted of 150 biphasic pulses, $0.2 \mathrm{~ms}$ phase width, $300 \mathrm{~Hz}$ pulse frequency, and $60 \mu \mathrm{A}$ current amplitude. We set the number of pulses and the pulse frequency based on previous work that showed the effectiveness of long-train, high frequency stimulation, in evoking movements that recapitulate ethologically relevant behavior (Graziano et al., 2002; Stepniewska et al., 2005; Baldwin et al., 2017). Although evoking movements was not our objective for ICMS+ISOI, we reasoned that the same stimulation parameters would effectively drive intrinsic M1 networks.

\section{Experiments on ICMS parameters}

The effects of ICMS parameters on activation maps were tested in a separate set of experiments. A range of microelectrode depths (200, 400, 1000,1400 , and $1800 \mu \mathrm{m}$ from cortical surface), current amplitudes (20, $40,60 \mu \mathrm{A})$, train durations $(18,36,75$, and 150 pulses), and pulse frequencies $(37,75,150$, and $300 \mathrm{~Hz})$, were tested. Other parameters were consistent with the previous section.

\section{Image analysis \\ Optical maps}

ISOI data were analyzed using custom MATLAB scripts. Two image subtractions were conducted as a pre-processing step to minimize global signals (e.g., cortical pulsations, blood vessels, illumination noise). (1) First-frame subtraction. In every trial, the first data frame was subtracted from subsequent frames. (2) Blank subtraction. For each block of trials, the frames of the blank condition were subtracted from the frames of the ICMS condition. After this pre-processing step, an average frame was calculated from consecutive frames that spanned 400-600 ms of image acquisition. Frame selection was optimized to coincide with peak reflectance change near the tip of the stimulating microelectrode, which typically occurred $1500-2000$ ms from stimulation onset. Finally, the mean frames calculated from the trials of a given condition were averaged together to generate maps for each ICMS condition minus blank condition.

Subtraction maps were then processed to aid visualization. Maps were convolved with a high-pass median filter (kernel $=150-250$ pixels) to correct uneven illumination and residual motion artifact. Maps were also convolved with a low-pass Gaussian filter (kernel $=10$ pixels) for smoothing. To enhance contrast, the distribution of pixel values within a map was clipped to $\pm 1.5 \mathrm{SD}$ from the median pixel value. In the final subtraction maps, dark pixels indicate decreased reflectance of red light, which is attributed to a hemodynamic response that ensues as a result of local increases in neural activity. Dark pixels therefore report cortical locations where ICMS evoked a response.

\section{Activation maps}

Activation maps were generated to objectively determine which pixels darkened in response to stimulation. Individual trials from the ICMS condition and the blank condition were first-frame subtracted and spatially convolved as described above for optical maps. However, in the present analysis, mean data frames were calculated separately for ICMS and blank conditions. Mean frames from the ICMS condition were then compared on a pixel-by-pixel basis to the mean frames from the blank condition. Pixels were considered active in response to stimulation if they were significantly darker $(t$ test, $p<0.001$ ) in the ICMS condition as compared with the blank condition.

\section{Intrinsic signal time course}

Time courses were measured from several regions of interest (ROIs; circle, $\sim 15$ pixel radius, $\sim 0.13 \mathrm{~mm}^{2}$ ). Pixel values within an ROI were averaged to a single value for each data frame. Values from successive data frames constituted the time course of a trial. Time courses from dozens of trials (same condition) were averaged to obtain the time course for a given ROI.

\section{Activation map quantification}

The activation map from each M1 stimulation site was quantified in relation to the motor map. First, the activation map was co-registered with the motor map. Next, the activation map was quantified by assigning every pixel to the spatially coincident zone within the motor map (i.e., arm zone, hand zone, etc.). The number of pixels in each zone was then converted to surface area $\left(\mathrm{mm}^{2}\right)$. Finally, the spatial range of the activation map was quantified according to the Euclidean distance $(\mathrm{mm})$ between the stimulation site and every pixel activated in response to stimulation.

\section{Stimulation site identity}

Blood vessels were used as landmarks to guide placement of the stimulating microelectrode into target sites within the motor map. Stimulation site identity was also confirmed from the activation map. Specifically, the stimulation site was presumed to be in the center of the cluster of pixels that was first to darken; typically, after $200 \mathrm{~ms}$ from stimulation onset. In most cases, the pertinent cluster of pixels was within $100 \mu \mathrm{m}$ of the stimulation site location noted during the experiment. The stimulation site was then classified (i.e., arm site, hand site, etc.) according to the motor map zone that contained the cluster of dark pixels (e.g., shoulder site, digit site, etc.). In mixed forelimb sites the cluster of dark pixels overlapped with arm and hand zones.

\section{Activation map alignment}

Activation maps from the same hemisphere were aligned to a common reference frame. Alignment was performed by estimating a projective transformation from points that were present in the blood vessel patterns of the ICMS condition, blank condition, and the common reference frame (estimateGeometricTransform function in MATLAB). The projective transformation produced a transform matrix that included scaling, rotation, and offset necessary to align each data frame to the common reference frame.

\section{Statistical analyses}

All statistical tests were performed using SPSS or MATLAB. In all instances, parametric tests were used if pertinent assumptions (e.g., normality and homoscedasticity) were not violated. Normality was tested using the Kolmogorov-Smirnov test and homoscedasticity was tested using Levene's test. Non-parametric tests were used if assumptions of parametric tests were not satisfied. For comparisons between two populations, either a two-sample $t$ test or a Wilcoxon rank-sum test was performed. For comparisons across more than two populations a one-way ANOVA was used with a post hoc Tukey's HSD test. For all tests, a 
significance level of $\alpha=0.05$ was used. In all figures, levels of significance are reported as follows: ${ }^{*} p<0.05,{ }^{* *} p<0.01$, ${ }^{* * *} p<0.001$

\section{Results}

We investigated the intrinsic connectivity of the forelimb representation in M1. In three squirrel monkeys, we first mapped the organization of the M1 forelimb representation using ICMS (Fig. 1A-F). Next, we measured connectivity for 64 sites within the M1 forelimb representation (Fig. 1D-F, red dots; Table 1). At every site, the ICMS evoked cortical response was recorded with ISOI (Fig. 1G). Coregistering the imaging results with the motor maps revealed two consistent features that likely reflect organizational principles of intrinsic M1 connectivity. First, every stimulation site activated several patches within the M1 forelimb representation. Second, patches mostly coincided with forelimb zones of the same functional classification as the stimulation site.
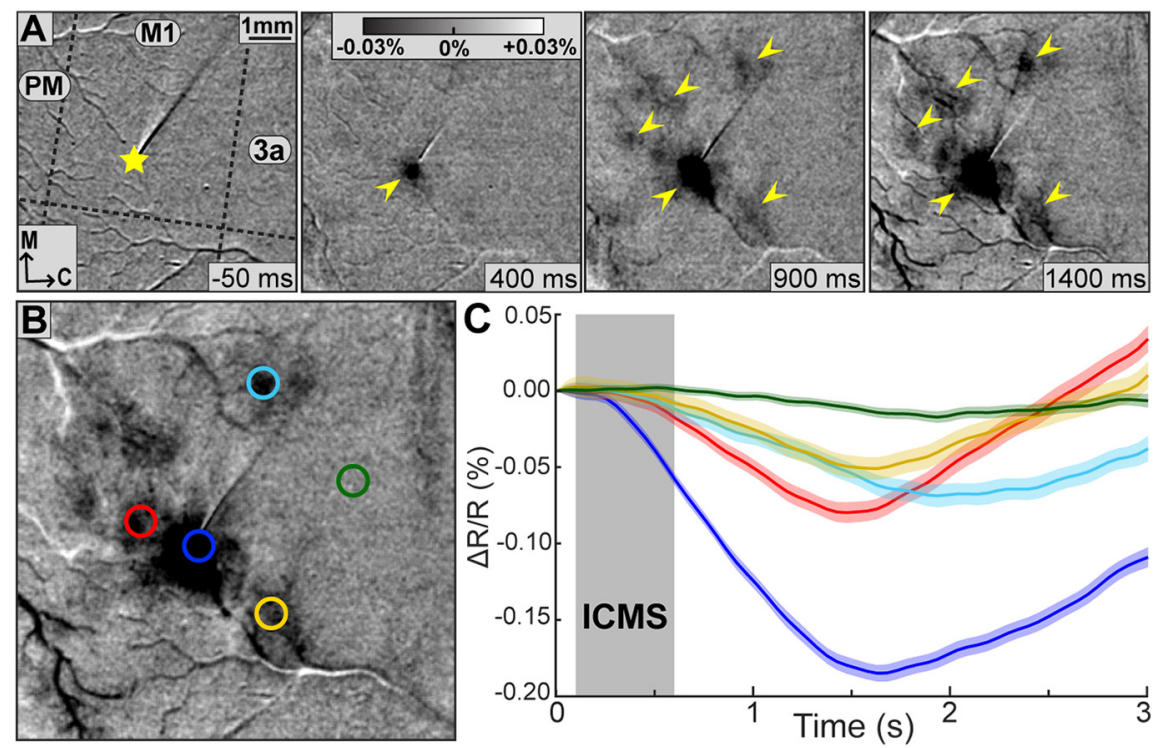

Figure 2. ICMS activates patches in the M1 forelimb representation. $\boldsymbol{A}$, Optical images: ICMS condition minus blank condition (50 trials/condition). Frames were temporally binned $20 \mathrm{~Hz}$ (50 ms/frame). Each panel is an average optical image after firstframe subtraction, spatial filtering, and clipping (median $\pm 1.5 \mathrm{SD}$ ). Time (ms) in relation to ICMS onset is in the bottom righthand corner of each frame. ICMS train (500-ms duration) started after two frames (100 ms) of baseline imaging. Yellow star marks the stimulation site, but the tip of the microelectrode was $1000 \mu \mathrm{m}$ below the cortical surface. The main activation patch (upward arrowhead) had the earliest onset. More distant patches (downward arrowheads) lagged the development of the main patch. Scale bar and reflectance intensity scale apply to all optical images. $\boldsymbol{B}$, Average optical image generated from the 10 frames acquired 1050-1550 ms from ICMS onset. Colored circles are ROls for time course analysis. C, Line plot shows time course of reflectance change (mean $\pm \mathrm{SEM}$ ). Line colors correspond to ROl colors in $\boldsymbol{B}$.

\section{Consistent organization of the M1 forelimb representation}

The M1 forelimb representation was organized consistently in the three squirrel monkeys. Digit and wrist zones were near the center of the M1 forelimb representation and were surrounded by elbow and shoulder zones (Fig. $1 A$ ). This topography was particularly apparent once shoulder and elbow zones were classified as arm and digit and wrist zones were classified as hand (Fig. 1C-F). A similar horseshoe/nested organization has been reported for the M1 forelimb representation in macaques (Kwan et al., 1978; Sessle and Wiesendanger, 1982; Park et al., 2001) and to some extent in New World squirrel monkeys (Dancause et al., 2008). However, a fractured organization is perhaps more widely recognized for New World monkeys (Strick and Preston, 1982; Gould et al., 1986; Donoghue et al., 1992; Nudo and Milliken, 1996). Current thresholds for evoking forelimb movements (median $=19.0 \mu \mathrm{A}$ ) were in the range expected for motor mapping under ketamine sedation (Nudo et al., 1996). Current thresholds were slightly lower for hand sites (median $=15.5 \mu \mathrm{A}$ ) as compared with arm sites $($ median $=21.0 \mu \mathrm{A})$. Rostral and caudal M1 borders were drawn near sites with current thresholds $>80 \mu \mathrm{A}$ (Fig. 1B).

Motor maps were built up in each animal over several experiments (30-60 ICMS sites/experiment). To assess the stability of ICMS-evoked movements over time, we retested a subset of forelimb sites months after initial motor mapping ( $n=56$ sites, 220 $\pm 152 \mathrm{~d}$ apart, mean $\pm 1 \mathrm{SD})$. The present analysis only includes sites in which the microelectrode location during retesting was within $250 \mu \mathrm{m}$ of the original site. In five squirrel monkeys (three cases in Fig. 1; two cases from a separate study), we found that $47 / 56(\sim 84 \%)$ of the retested sites had the same classification at both time points. Results from this sample of retested sites suggest that motor maps were relatively stable over time.

\section{ICMS activates patches in the M1 forelimb representation}

To investigate connectivity, ICMS trains ( 150 biphasic pulses, 0.2 ms pulse width, $300 \mathrm{~Hz}, 60 \mu \mathrm{A}$ ) were delivered $1000 \mu \mathrm{m}$ below the cortical surface. The evoked cortical response was measured with ISOI. The average response for each site was determined from 50 ICMS trials. The rationale here is that ICMS would modulate intrinsic signal levels in M1 zones that are connected to the stimulation site (Fig. 1G). Indeed, ICMS onset immediately drove a decrease in reflectance (i.e., pixel darkening) in well-defined zones ( 0.25 to $1.0 \mathrm{~mm}$ radius) that we refer to as patches. Quantifying the patches from dozens of sites in the M1 forelimb representation (Fig. $1 D-F$ ) was the linchpin for uncovering the principles that govern intrinsic M1 connectivity.

Figure 2 shows intrinsic signal time courses from a representative site. The earliest decrease in reflectance occurred at the stimulation site (Fig. 2A, upward arrowhead). This patch was evident within $400 \mathrm{~ms}$ of ICMS onset and it expanded over time to peak in size $(\sim 1 \mathrm{~mm}$ radius $)$ by $1400 \mathrm{~ms}$ from ICMS onset. Beyond this main patch, at least four smaller patches were evident within the M1 forelimb representation (Fig. 2A, downward arrowheads). Activation in distant patches lagged the main patch (Fig. 2A, 400 and $900 \mathrm{~ms}$ ). We interpret main and distant patches as zones with connectivity, most likely horizontal connections, to the stimulation site. For each stimulation site, we consider the patches and the connections that link them as an intrinsic network within the M1 forelimb representation.

\section{Intrinsic signal time course}

To quantify the time course of the evoked cortical response, reflectance change was measured in 5 ROIs (Fig. $2 B$, colored circles). In the four ROIs that overlapped patches, reflectance started to decrease within 200-400 ms of ICMS onset. The earliest and most intense reflectance decrease was in the center of 

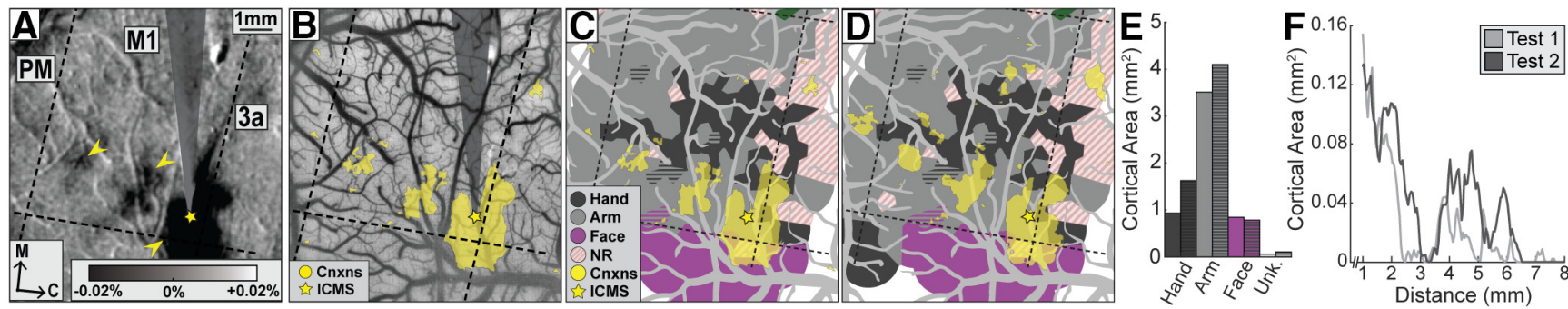

Figure 3. Activation maps are stable over months. A, Average optical image: ICMS condition minus blank condition ( 50 trials/condition). Average was generated from three frames (200 ms/ frame) acquired 1000-1600 ms from ICMS onset. Gray triangle masks the stimulating microelectrode and yellow star depicts the stimulation site. Upward arrowhead points to the main activation patch and downward arrowheads point to distant activation patches. $\boldsymbol{B}$, Yellow pixels were significantly darker in the ICMS condition as compared with the blank condition $(t$ test, $\mathrm{df}=98$, $p<0.001$ ). Yellow pixels constitute the activation map of the stimulation site. C, Co-registration of the activation map with the motor map shows that most yellow pixels spatially coincided with the arm representation (light gray). $\boldsymbol{D}$, Activation map acquired $280 \mathrm{~d}$ after the test in $\boldsymbol{C}$. Stimulation sites in $\boldsymbol{C}, \boldsymbol{D}$ were separated by $193 \pm 19 \mu \mathrm{m}$ and there was $74.3 \%$ overlap between their respective activation maps. Note, motor maps in $\boldsymbol{C}, \boldsymbol{D}$ are reproductions of the same map. $\boldsymbol{E}$, Quantification of the activation maps in relation to the motor map. Tests 1 and 2 are from $\boldsymbol{C}$, $D$, respectively. Yellow pixels that overlapped zones not mapped with ICMS, or zones that did not respond to ICMS, are classified as Unknown. $\boldsymbol{F}$, Spatial distribution of the activations maps as a function of distance from stimulation site.

the main patch (Fig. $2 B$, dark blue). Reflectance change in this ROI peaked $\sim 1500 \mathrm{~ms}$ after ICMS onset (Fig. $2 C$, dark blue). At the periphery of the main patch (Fig. $2 B, C$, red), reflectance change was $\sim 50 \%$ less intense than in the center of the main patch ( $\sim 1 \mathrm{~mm}$ away). In distant patches (Fig. $2 B, C$, cyan and yellow), reflectance change was slightly less intense than in the periphery of the main patch. Lack of modulation in the control ROI (Fig. 2B,C, green) supports the spatial specificity of the ICMS evoked cortical response. The present time courses were consistent across dozens of sites that we investigated in M1 and in somatosensory cortex. Time courses for the main patch were also consistent with previous studies using ICMS+ISOI in posterior parietal cortex and in somatosensory cortex (Stepniewska et al., 2011; Brock et al., 2013).

In addition to informing us on M1 connectivity, we investigated intrinsic signal time courses to set timing parameters. In 1 experiment, image acquisition was purposely extended to $20 \mathrm{~s} /$ trial to ensure the capture of the late phases of intrinsic signal modulation. In addition, alternate trials were imaged with red $(625 \mathrm{~nm})$ or with green illumination $(528 \mathrm{~nm})$ to ensure that intrinsic signal modulations related to oximetry and to blood volume were both taken into consideration. Observations from this experiment motivated us to set image acquisition to $4 \mathrm{~s}$ to include at least $1 \mathrm{~s}$ of data after the largest/slowest signals peaked. We set the intertrial to $12 \mathrm{~s}$, which is when the same signals approached baseline.

\section{Activation maps are stable over months}

In several stimulation sites, we investigated whether activation maps were reproducible over months. One of those stimulation sites was in the arm zone near the face representation (Fig. 3). In the average optical map (ICMS minus blank), the main patch (Fig. $3 A$, upward arrowhead) was approximately centered on the stimulation site (Fig. $3 A$, yellow star). The M1/3a border roughly bisected the main patch. Two smaller patches were rostral to the main patch (Fig. $3 A$, downward arrowheads). All patches corresponded closely with pixels that were statistically darker in the ICMS condition as compared with the blank condition ( $t$ test, $p<0.001$; Fig. $3 B$, yellow pixels). Thus, the yellow pixels that comprised the activation map effectively report the locations of patches with connectivity to the stimulation site. After co-registering the activation map with the motor map (Fig. $3 C$ ), it was evident that parts of the main patch overlapped the M1 arm zone and the M1 face representation. In contrast, distant patches were almost exclusively in the M1 arm zone. Retesting the same stimulation site after $280 \mathrm{~d}$ showed that the overall organization of the activation map was conserved (Fig. 3D). Distant patches were more apparent in the second test, potentially due to differences in site location (intersite distance $=193 \pm 19 \mu \mathrm{m}$ ) or state of animal. Nevertheless, overlap between the two activation maps was $74.3 \%$ in this particular example and $80.9 \pm 6.8 \%$ $($ mean $\pm 1 \mathrm{SD})$ in six retested sites [days between tests $=219 \pm$ $101 \mathrm{~d}$ (mean $\pm 1 \mathrm{SD})$; intersite distance $=125 \pm 50 \mu \mathrm{m}$ (mean \pm $1 \mathrm{SD}$ )]. Results from this sample of retested sites suggest that activation maps were stable over months, possibly longer.

To quantify the spatial organization of the activation maps, every pixel activated (Fig. 3C,D, yellow) was classified according to the M1 zone that it overlapped. Pixels were then converted to surface area $\left(13 \mu \mathrm{m}^{2} / \mathrm{pixel}\right)$. In the first test, the stimulation site activated $\sim 4 \mathrm{~mm}^{2}$ in the arm zone and $\sim 1.5 \mathrm{~mm}^{2}$ in the hand zone (Fig. $3 E$ ). The arm-to-hand ratio was comparable in the activation map of the second test. Only a small fraction of M1 activation $\left(<1.0 \mathrm{~mm}^{2}\right)$ overlapped the face representation. It is important to note that the stimulation sites in both tests were $\leq 1$ $\mathrm{mm}$ from the forelimb/face border. At such close distance, the near absence of activation in the face representation supports the likelihood that patches were driven by horizontal connections that did not cross from the forelimb representation into the face representation (Huntley and Jones, 1991; Weiss and Keller, 1994). We reached the same conclusion by stimulating a site in the M1 face representation that was no more than $1.75 \mathrm{~mm}$ from the lateral arm sites (Fig. 1D). That site activated patches in the face representation that covered more than four times the surface area as the activation patches in the forelimb representation.

To quantify the spatial range of the activation maps, distance was measured between the stimulation site and every activated pixel. Spatial ranges were relatively consistent between the first and second tests. Overall, surface area of activation decayed with distance from the stimulation site (Fig. 3F). Nevertheless, peaks and valleys in the line plots show that activation patches were punctuated by zones with no evoked activation. The present activation pattern supports the organization proposed in (Fig. $1 G$, bottom) and argues against a strict monotonic decline in connectivity as a function of distance (Fig. 1G, top).

\section{Arm and hand zones have distinctive activation maps}

Our next objective was to examine whether arm and hand zones have the same connectivity patterns. If they do, then we would 

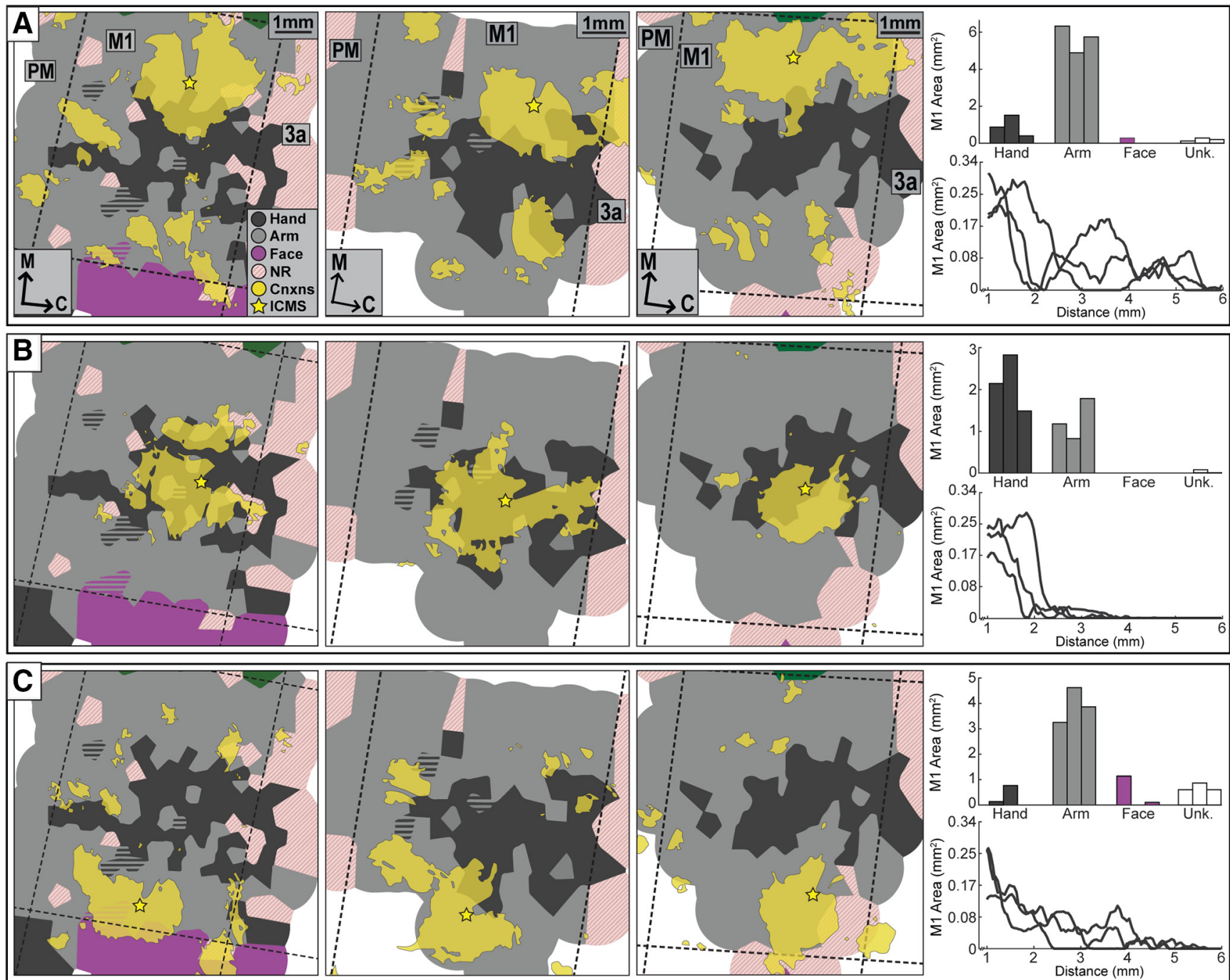

Figure 4. Arm and hand zones have distinctive activation maps. Activation maps from nine stimulation sites (three sites/monkey) co-registered with respective motor maps. Yellow stars depict stimulation sites. Yellow pixels were significantly darker in the ICMS condition as compared with the blank condition ( $t$ test; $p<0.001)$. A, Activation maps (one map/monkey) for stimulation sites in medial aspects of the arm zone. Activation maps had consistent patterns across animals despite small differences in the locations of the stimulation sites. Bar plot shows that activation was primarily in the arm zone. Line plot shows the spatial range of the three activation maps as a function of distance from stimulation sites. B, Activation maps evoked from stimulation in the hand zone. Bar plot and line plot follow the same format in $\boldsymbol{A}$. C, Activation maps in response to stimulation sites in lateral aspects of the arm zone. Note that the bar and line plots in $\boldsymbol{B}$ were clearly different from those in $\boldsymbol{A}, \boldsymbol{C}$.

expect activation maps to have the same spatial configuration across stimulation sites regardless of their location within the M1 forelimb representation. In contrast, if connectivity patterns are governed by motor map somatotopy, then we would expect activation maps to have distinctive patterns for stimulation sites in arm and hand zones. To evaluate these competing frameworks, we compared activation maps from stimulation sites in (1) medial arm zones (Fig. 4A), (2) hand zones (Fig. 4B), and (3) lateral arm zones (Fig. 4C). For each zone, we compared one stimulation site/monkey.

A main activation patch ( $\sim 1 \mathrm{~mm}$ radius) surrounded each of the nine stimulation sites (Fig. 4; one yellow star/panel). This is consistent with Figures 2, 3 and the dozens of sites that we tested, which suggests that the main patch reflects a fundamental feature of M1 connectivity. Dense, isotropic, horizontal connections (1 $\mathrm{mm}$ ) are the most likely driver of the main patch. Beyond the main patch, activation maps varied according to the forelimb zone stimulated. Stimulation in medial arm zones activated several distant patches that overlapped primarily with arm zones
(Fig. 4A, yellow patches). Quantifying the surface area of the patches confirmed a $\sim 3$-to-1 arm-hand distribution that was consistent across animals (Fig. 4A, bar plot). Line plots show the long spatial range (up to 5-6 $\mathrm{mm}$ ) of these activation maps and their relative consistency across animals (Fig. 4A). Stimulation in lateral arm zones evoked activation maps (Fig. 4C) that were akin to mirror images of the activation maps in Figure $4 A$. Similarities between the two sets of activation maps were also evident in the arm-hand distribution of patches (Fig. 4A,C, bar plots).

Stimulation in hand zones evoked activation maps that differed in several ways from those evoked from stimulation in arm zones. First, patches evoked from hand sites were concentrated in the center of the forelimb representation (Fig. 4B). Second, patches overlapped primarily with hand zones (Fig. $4 B$, bar plot). Third, distant patches were much closer to the main patch and in many instances fused with the main patch. This was evident in the limited $(\sim 2-3 \mathrm{~mm})$ spatial range of the activation maps (Fig. $4 B$, line plot). Thus, arm and hand sites evoked activation maps 

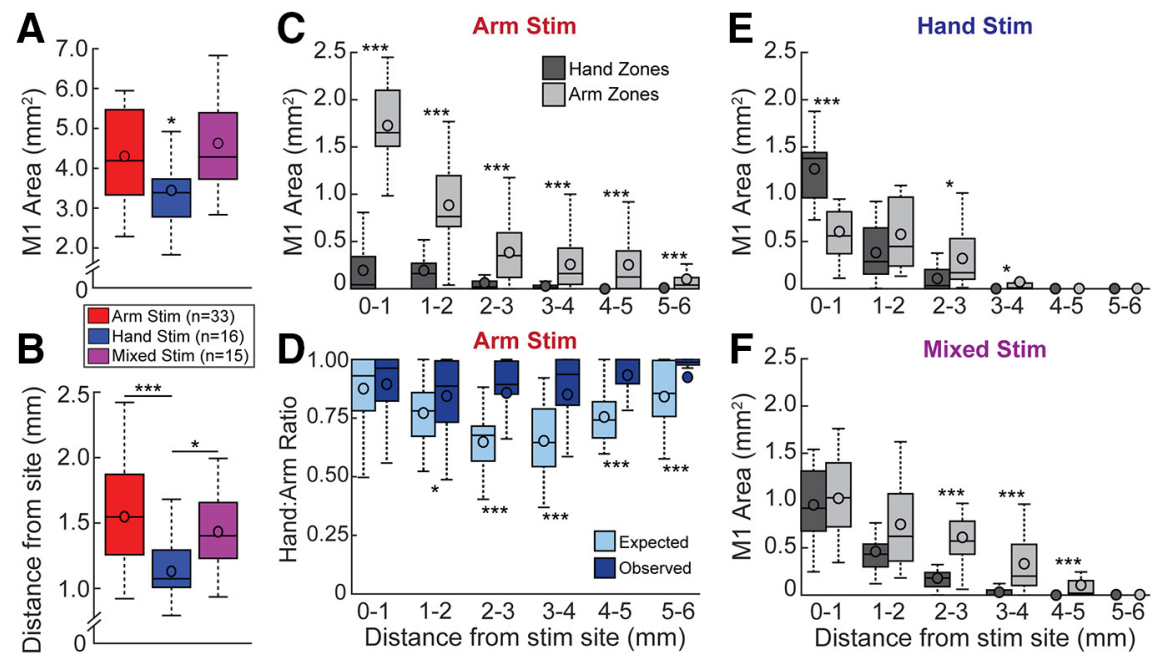

Figure 5. Population of M1 sites confirm differential connectivity for arm and hand zones. $\boldsymbol{A}$, Size of activation maps evoked from stimulation in arm sites, or hand sites, or mixed sites. $\boldsymbol{B}$, Spatial range of activation maps computed from the average distance between ICMS sites and pixels that constitute the activation maps; ${ }^{*} p<0.05,{ }^{* * *} p<0.001$ (Tukey's HSD test). $\boldsymbol{C}$ Contrasting the spatial range of the activation maps evoked from stimulation in arm and hand sites; $1 \mathrm{~mm}$ intervals are referenced from the stimulation sites. Independent comparisons were conducted at every interval (Wilcoxon rank-sum test, 0-1 mm: $Z=-6.90, p=5.4 \times 10^{-12} ; 1-2 \mathrm{~mm}: Z=-6.11, p=1.0 \times 10^{-9} ; 2-3 \mathrm{~mm}: Z=-4.81, p=1.5 \times 10^{-6} ; 3-4 \mathrm{~mm}:$ $\left.Z=-4.87, p=1.1 \times 10^{-6} ; 4-5 \mathrm{~mm}: Z=-4.55, p=5.4 \times 10^{-6} ; 5-6 \mathrm{~mm}: Z=-5.06, p=4.2 \times 10^{-7}\right)$. D, At every interval, the surface area activated (observed) was directly compared with the surface area that could have been expected (expected) from the arm-to-hand ratios (Wilcoxon rank-sum test, $0-1 \mathrm{~mm}: Z=-0.45, p=0.66 ; 1-2 \mathrm{~mm}$ : $Z=-2.20$, $p=0.03 ; 2-3 \mathrm{~mm}: Z=-5.25, p=1.5 \times 10^{-7} ; 3-4 \mathrm{~mm}: Z=-4.75, p=2.1 \times 10^{-6} ; 4-5 \mathrm{~mm}: Z=-4.91, p=9.3$ $\times 10^{-7} ; 5-6 \mathrm{~mm}: Z=-3.79, p=1.5 \times 10^{-4}$ ). $\boldsymbol{E}$, Same as $\boldsymbol{C}$, but for hand sites (Wilcoxon rank-sum test, $0-1 \mathrm{~mm}$ : $Z=3.86, p=1.1 \times 10^{-4} ; 1-2 \mathrm{~mm}: Z=-1.64, p=0.10 ; 2-3 \mathrm{~mm}: Z=-2.24, p=0.03 ; 3-4 \mathrm{~mm}: Z=-2.22, p=$ 0.03). $\boldsymbol{F}$, Same as $\boldsymbol{C}$, but for mixed sites (Wilcoxon rank-sum test, $0-1 \mathrm{~mm}: Z=-0.46, p=0.65 ; 1-2 \mathrm{~mm}: Z=-1.53$, $p=0.13 ; 2-3 \mathrm{~mm}: Z=-3.57, p=3.6 \times 10^{-4} ; 3-4 \mathrm{~mm}: Z=-3.51, p=4.5 \times 10^{-4} ; 4-5 \mathrm{~mm}: Z=-4.19$, $\left.p=2.7 \times 10^{-5}\right)$.

with distinctive spatial configurations, which suggests that intrinsic M1 connectivity is closely linked to the somatotopy of the forelimb representation. This observation does not support the possibility of a universal intrinsic M1 network that simply repeats throughout the forelimb representation.

\section{Population of M1 sites confirm differential connectivity for arm and hand zones}

Activation maps from a population of M1 sites (64 total, 33 arm, 16 hand, and 15 mixed) were quantified with the same approach used for the representative sites in Figure 4. Mixed sites were in zones that evoked both arm and hand movements. Activation maps differed in size between the three site classifications (Fig. $5 A$; ANOVA, $\left.F_{(2,61)}=4.65, p=0.013\right)$. Hand sites activated the smallest maps $\left(\right.$ mean $=3.45 \mathrm{~mm}^{2}$ ) as compared with arm sites (mean $=4.30 \mathrm{~mm}^{2}$, Tukey's HSD test, $p=0.043$ ) and to mixed sites (mean $=4.63 \mathrm{~mm}^{2}$, Tukey's HSD test, $p=0.015$ ). Similarly, the spatial range of the activation maps differed between the three site classifications (Fig. $5 B$; ANOVA, $F_{(2,61)}=7.63, p=$ $0.001)$. Spatial ranges were shorter for hand sites (mean $=1.13$ $\mathrm{mm}$ ) as compared with arm sites (mean $=1.55 \mathrm{~mm}$, Tukey's HSD test, $p=0.001$ ) and to mixed sites (mean $=1.45 \mathrm{~mm}$, Tukey's HSD test, $p=0.043$ ). Collectively, the results show that activation maps from hand sites were small and spatially restricted as compared with arm sites and mixed sites. Thus, results from the population of M1 sites support observations (Fig. 4) of distinctive connectivity patterns for arm and hand zones.

Our next objective was to quantify the activation maps in relation to the motor maps. To that end, the surface area activated in arm and hand zones was measured using the same approach in Figures 3, 4. We then compared the amount of surface area activated in hand and arm zones (Wilcoxon rank-sum test) at $1 \mathrm{~mm}$ intervals from the stimulation site. In all three site classifications, activation maps decreased in size with distance from the stimulation site (Fig. 5C,E,F). In addition, the extent to which activation maps overlapped with arm and hand zones depended on the motor output of the stimulation site. These results further support the likelihood that arm and hand zones are endowed with distinctive connectivity patterns and do not conform to one connectivity motif.

\section{Arm sites}

Only arm sites activated patches as far as $5-6 \mathrm{~mm}$ from stimulation site. This was consistent with observations that arm sites had the longest connectivity range (Fig. 5B). Also, at every distance interval from the stimulation site, more surface area was activated in arm zones as compared with hand zones (Fig. 5C). We investigated whether this size difference could have simply resulted from the M1 forelimb representation having larger arm zones than hand zones (Fig. 1). We reasoned that observed activation (i.e., actual size of patches) would not be different from expected activation (i.e., hypothetical size of patches) in the event that activation maps simply reflect size differences between arm and hand zones in the forelimb representation. Thus, for a given activation map, at every $1 \mathrm{~mm}$ interval from the stimulation site, we calculated the ratio of surface area activated within the arm zone (i.e., observed activation): [area activated in arm zone/(area activated in hand zone + area activated in arm zone)].

Similarly, for a given motor map, at every $1 \mathrm{~mm}$ interval from the stimulation site, we calculated the ratio of surface area of the arm zone (i.e., expected activation): [area of arm zone/(area of hand zone + area of arm zone)].

Finally, we compared the observed activation to the expected activation at $1 \mathrm{~mm}$ intervals (Wilcoxon rank-sum test). With the exception of the first interval, we found that the observed activation was consistently larger than the expected activation (Fig. $5 D$ ). Thus, more surface area was activated in the arm zone than could be expected from the topography of the forelimb representation. Similarly, less surface area was activated in the hand zone than could be expected from the topography of the forelimb representation. Discrepancy between observed and expected activation supports the likelihood of spatially selective connectivity for sites in the arm zone.

\section{Hand sites}

Unlike arm sites, hand sites did not activate patches beyond 3-4 $\mathrm{mm}$ from stimulation (Fig. $5 E$ ). In the first $1 \mathrm{~mm}$ interval, more surface area was activated in hand zones as compared with arm zones. Beyond the first interval, activation was distributed evenly between arm and hand zones (1-2 $\mathrm{mm})$, or overlapped more 
with arm zones (2-4 mm). Nevertheless, the ratio of arm-hand activation (observed activation) did not differ from the ratio of arm-hand zones (expected activation) in the motor map.

\section{Mixed sites}

Activation maps here were in between activation maps from arm and hand sites. Patches were present as far as $4-5$ $\mathrm{mm}$ from stimulation (Fig. $5 F$ ). Within $2 \mathrm{~mm}$ of stimulation, the area activated was comparable between arm and hand zones. However, beyond $2 \mathrm{~mm}$, activation was almost entirely in arm zones. Like hand sites, observed activation and expected activation (data not shown) were not different for mixed sites.

\section{Activation maps recapitulate the forelimb representation}

We sought to visualize the activation maps for the populations of arm and hand sites. From the results in Figure 5, we reasoned that superimposing activation maps from arm sites would lead to patches overlapping primarily with arm zones (Fig. 6A,D,G, light gray). Similarly, we expected activation maps from hand sites to overlap mostly with hand zones (Fig. 6A,D,G, dark gray). Indeed, in all three monkeys, arm sites activated patches that were concentrated in arm zones and largely avoided the hand zones in the center of the forelimb representation (Fig. 6B,E,H). This spatial pattern was most evident in Figure $6 B$, which had the largest number of stimulation sites in the arm zone. In contrast, hand sites activated patches that were most densely concentrated in the hand zone and had limited overlap with the arm zone (Fig. 6C,F,I). Thus, the activation maps from stimulation in arm sites and hand sites recapitulate the topography of the forelimb representation.

\section{Selective overlap between activation maps}

We investigated the relationship between site classification and spatial coincidence of M1 networks. We compared overlap in activation maps between all matching pairs (i.e., pairs of arm sites and pairs of hand sites) and all non-matching pairs (i.e., one arm site and one hand site). For matching pairs and non-matching pairs, overlap between activation maps declined with intersite distance (Fig. 7A; 418 total pairs across three animals). This was consistent with our observation that surface area of activation decreased with distance from stimulation (Figs. 4, 5). In addition, at every distance interval, activation maps from matching pairs had more overlap than activation maps from non-matching pairs (Wilcoxon ranksum test; Fig. 7A). For example, several overlapping patches were observed from a matching pair of sites on opposite sides of the arm zone (Fig. $7 B$, red). In contrast, activation maps from an arm site and a hand site had nearly no overlap (Fig. 7C) despite closer intersite distance as compared with the matching pair (Fig. 7B). Thus, the results collectively show that networks that control the same forelimb segment overlap more with each other than with networks that control other segments of the forelimb.

\section{Reciprocal connectivity between patches of an activation map}

Our next objective was to examine the spatial selectivity of the networks that link activation patches. To that end, we used one activation map as a reference (Fig. $7 D$, green) for planning four stimulation sites (Fig. $7 D$, yellow stars) that would yield four additional activation maps (Fig. $7 E-H$ ). Sites $\mathrm{E}$ and $\mathrm{G}$ targeted patches of the reference map (Fig. 7D). Both stimulation sites activated at least one distant patch that spatially coincided with the location of the reference stimulation site (Fig. 7E,G, coincidence of green star with yellow patch). For direct comparison, sites $\mathrm{F}$ and $\mathrm{H}$ were purposely placed to avoid patches from the reference map (Fig. 7D). Moreover, sites $\mathrm{F}$ and $\mathrm{H}$ were matched with sites $E$ and $G$ with respect to distance from the reference site. None of the patches from sites $\mathrm{F}$ or $\mathrm{H}$ overlapped with the vicinity of the reference site (Fig. $7 F, H$ ). We extended the present analysis to all site pairs with an intersite distance $\geq 2 \mathrm{~mm}$. The relationship observed between reference and stimulation sites in Figure $7 E, G$ was present in $7 / 10$ site pairs (intersite distance $=$ $2820 \pm 620 \mu \mathrm{m}$, mean $\pm 1 \mathrm{SD})$. Similarly, the relationship observed between reference and stimulation sites in Figure $7 F, H$ was present in $177 / 188$ (intersite distance $=2950 \pm 740 \mu \mathrm{m}$, 

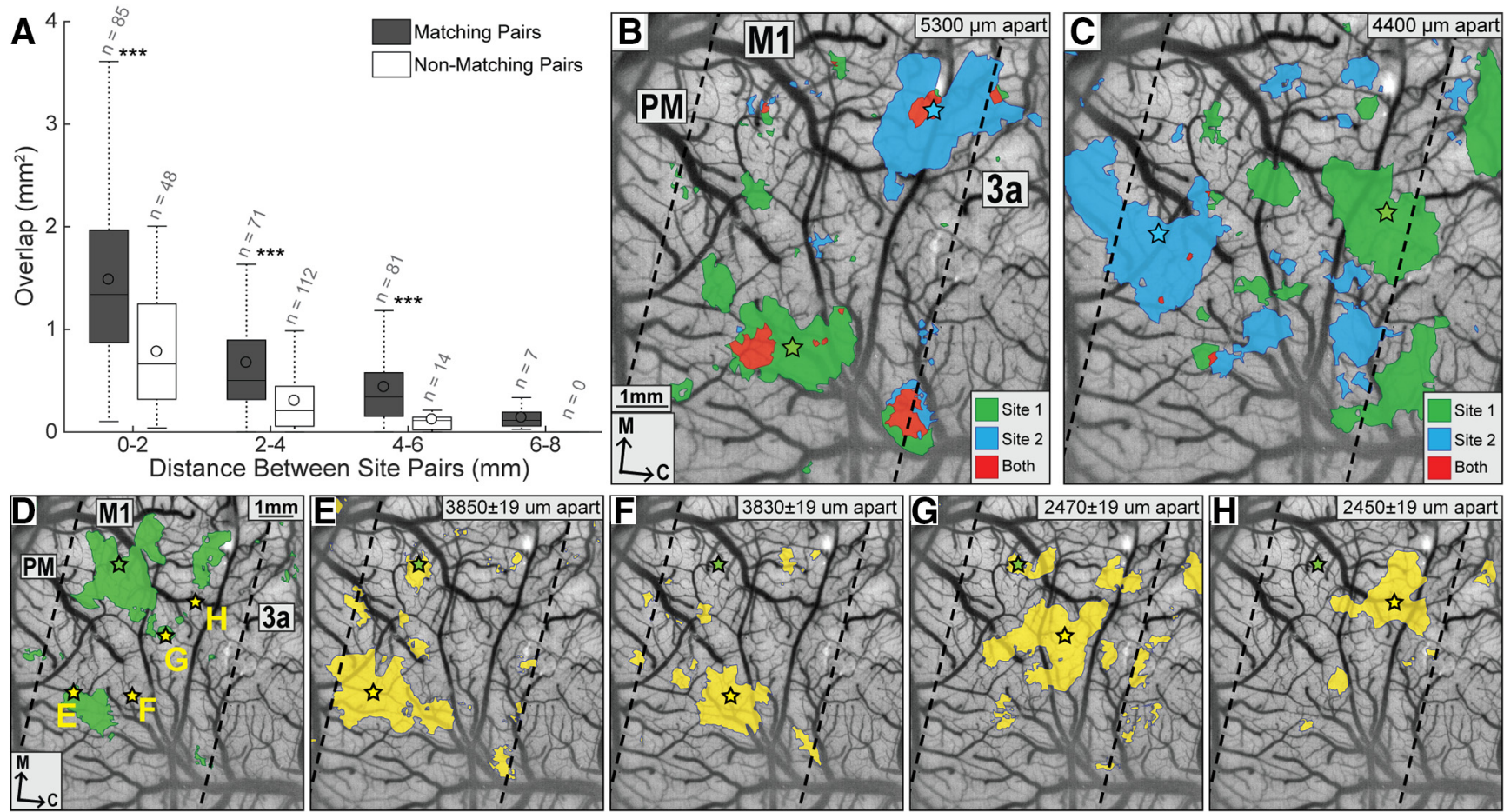

Figure 7. Overlap between activation maps is related to site identity and intersite distance. $A$, Spatial overlap between pairs of activation maps (418 comparisons; 3 animals). Comparisons are grouped according to intersite distance and whether sites were functionally matching (e.g., two arm sites) or non-matching (i.e., one arm site and one hand site). Overlap between activation maps declined with intersite distance. Overlap was consistently greater for matching sites than for non-matching sites (Wilcoxon rank-sum test, $0-2 \mathrm{~mm}$ : $Z=4.51$, $\left.p=6.4 \times 10^{-6} ; 2-4 \mathrm{~mm}: Z=5.82, p=5.9 \times 10^{-9}, 4-6 \mathrm{~mm}: Z=3.49, p=4.8 \times 10^{-4}\right)$. $\boldsymbol{B}$, Activation maps from a pair of matching sites. Stimulation sites (green and blue stars) were $5300 \mu \mathrm{m}$ apart. The two activation maps (green and blue patches) overlapped in several locations (red). C, Activation maps (blue and green) from a pair of non-matching sites (4400 $\mu \mathrm{m}$ apart) had almost no overlap (red). $\boldsymbol{D}$, Reference activation map (green patches) in response to stimulation at the green star. Yellow stars mark the locations of four stimulation sites. Sites $\boldsymbol{E}, \boldsymbol{G}$ were placed to overlap green patches. Sites $\boldsymbol{F}, \boldsymbol{H}$ were placed to avoid green patches. $\boldsymbol{E}-\boldsymbol{H}$, Activation maps evoked from corresponding stimulation sites in $\boldsymbol{D}$. For each activation map, stimulation was delivered in the location of the yellow star. Green stars are included for reference only. Intersite distance (yellow and green star) is in the top right corner of each activation map. Reciprocal connectivity (overlap between green star and yellow pixels) is evident in $\boldsymbol{E}$ and $\boldsymbol{G}$, but not in $\boldsymbol{F}$ or $\boldsymbol{H}$.

mean $\pm 1 \mathrm{SD}$ ). Thus, the results collectively indicate that patches that comprise an activation map are reciprocally connected with the reference site.

\section{Validation experiments}

Our results from dozens of stimulation sites confirm the effectiveness of ICMS+ISOI for mapping cortical connectivity. Nevertheless, mapping brain networks with ICMS+imaging (ISOI or fMRI) is relatively new and differs mechanistically from well-established neuroanatomical tracing methods. This motivated us to test ICMS+ISOI in well-defined cortical networks before using the approach to reveal the organization of the understudied M1 networks. Somatosensory cortical areas were an excellent testbed for several reasons. (1) Connections have been studied extensively with tracer injections. (2) Somatosensory maps can be exploited for spatial quantification of the activation maps. (3) Accessibility from the same cranial window used for M1.

\section{ICMS + ISOI connectivity is consistent with previous tracer injections}

Our objective was to determine if stimulation in representations of individual digits (areas $3 b$ and 1 ) would evoke activation maps comparable to connectivity patterns revealed with neuroanatomical tracers (Liao et al., 2013; Négyessy et al., 2013). In two monkeys ( $M$ and $G$ in Table 1), we first used microelectrode recordings to map somatosensory representations of the hand (Fig. 8A,B). Our maps were consistent with somatotopy long- established for New World monkeys (Merzenich et al., 1978; Kaas et al., 1979). Next, we investigated four somatosensory sites (two sites/monkey) using the same ICMS+ISOI parameters that were applied in M1.

For the first stimulation site in area $3 \mathrm{~b}$, the microelectrode was in the representation of the distal phalanx of the fourth digit (i.e., distal D4). The activation map was comprised of three patches (Fig. $8 C$ ). The main patch ( $\sim 1 \mathrm{~mm}$ radius) was approximately centered on the stimulation site (Fig. $8 C$, yellow star) and mostly overlapped distal D4 followed by distal D3. This patch also encroached into caudal aspects of area $3 \mathrm{a}$. The other two patches were located in area 1 where they overlapped D4 and D3. Similar activation patterns were obtained from a second stimulation site in area $3 \mathrm{~b}$ where the microelectrode was in proximal D1 (Fig. 8D). The main activation patch was located in area $3 \mathrm{~b}$ and surrounded the stimulation site (yellow star). Distant patches were smaller and confined to a narrow medio-lateral strip within area 1.

Next, we investigated two sites in area 1 . The first site activated a main patch that was elongated in the rostro-caudal direction and straddled areas $3 \mathrm{~b}$ and 1 (Fig. $8 E$ ). This patch was largely confined to proximal D4 and D3. Most of the activation in area $3 \mathrm{~b}$ likely belonged to a distant patch that fused with the main patch in area 1 . Smaller patches in areas $3 a$ and 2 were in the same medio-lateral strip as the main patch. The lateral patch in area 1 overlapped the palm. These results were confirmed in a second stimulation site in area 1 (Fig. $8 F$ ) where most of the activation was confined to the matching digit in area $3 \mathrm{~b}$. 

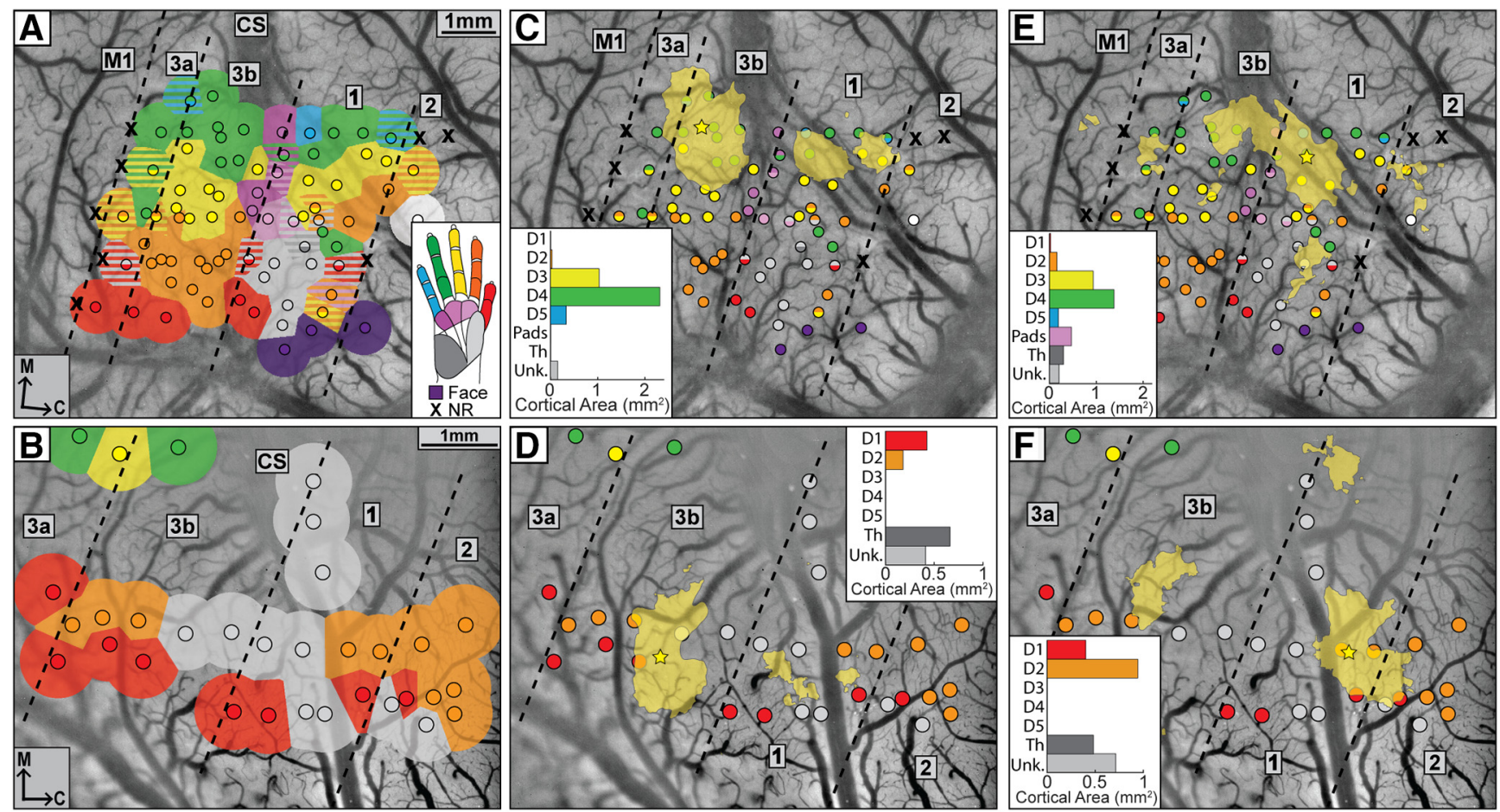

Figure 8. Somatosensory cortex connectivity is consistent with previous tracer injections. Top row, Monkey M. Bottom row, Monkey G. $\boldsymbol{A}$, Map of the somatosensory hand representation. Microelectrode recording sites (colored circles, $n=87$ ) were classified according to multiunit responses to receptive field mapping. Voronoi tiles ( $0.5 \mathrm{~mm}$ radius) are color-coded according to the hand illustration. Striped tiles are dual representations (one color/representation). Cortical borders (dashed lines) were estimated from receptive field mapping results. D1-D5: digits 1-5; Th: thenar. $\boldsymbol{B}$, Partial map of the somatosensory hand representations in monkey $\mathbf{G}(n=35$ sites). $\boldsymbol{C}$, Activation map (yellow patches) evoked in response to stimulation in a site (yellow star) in the representation of distal D4 in area 3b. Receptive field mapping sites are shown for reference. Bar plot shows the organization of the activation map in relation to the somatosensory map. Activation patches that overlapped unresponsive zones or unmapped zones are classified as "unknown." D, A similar activation map was evoked for a stimulation site in proximal D1 in area 3b. $\boldsymbol{E}$, Activation map for an ICMS site that straddled the representations of digits 3 and 4 in area 1. $\boldsymbol{F}$, Activation map for an ICMS site within digit 2 in area 1.

Activation maps from the four somatosensory sites corresponded closely with connection patterns revealed with neuroanatomical tracer injections into somatosensory representations of individual digits (Liao et al., 2013, their Fig. 7; Négyessy et al., 2013, their Figs. 7, 9). The near absence of activation patches in M1 and limited spread of activation patches in the medio-lateral direction ( $\leq 2$ digit representations) was also consistent with the tracer studies. Thus, our somatosensory results confirm the reliability of ICMS+ISOI for mapping cortical networks.

Activation maps are conserved across stimulation intensities Stimulation parameters have varied considerably across studies that investigated connectivity using ICMS+imaging (ISOI or fMRI). It was therefore important here to test how systematic variations in stimulation parameters might affect the evoked cortical response. We focused on microelectrode depth, current amplitude, train duration, and pulse frequency. Only one parameter was tested in each M1 site.

\section{Microelectrode depth}

We expected the most intense activation from depths in which the microelectrode was far enough from the cortical surface to at least reach layers $2 / 3(\sim 1000 \mu \mathrm{m}$ from surface). Five stimulation depths were tested $(200,600,1000,1400$, and $1800 \mu \mathrm{m})$ using the same ICMS parameters for M1 connectivity (150 biphasic pulses, $0.2 \mathrm{~ms}$ pulse width, $300 \mathrm{~Hz}, 60 \mu \mathrm{A}$ ). Locations of the main patch (Fig. 9A, yellow star) and the distant patches (arrowheads) were consistent across depths. However, distant patches grew in size with microelectrode depth. The effect was most evident $4-5 \mathrm{~mm}$ from the stimulation site (Fig. 9B,C) and could be attributed mostly to size differences in the patch near the border with area 3a (Fig. 9A). Distant patches in general appear to have been the primary factor in the step-wise increase in the size of the activation maps for stimulation depths 1000 and $1400 \mu \mathrm{m}$ (Fig. 9B). This is consistent with previous work showing that the longest horizontal connections within M1 originate in layer 5 (Aroniadou and Keller, 1993). That activation maps were comparable in size for stimulation depths 1400 and $1800 \mu \mathrm{m}$ supports the likelihood that horizontal connections, as opposed to white matter axons, were the main driver of the activation maps.

\section{Pulse amplitude}

Three pulse amplitudes were tested $(20,40,60 \mu \mathrm{A})$ while other ICMS parameters remained constant $(1000 \mu \mathrm{m}$ depth, 150 biphasic pulses, $0.2 \mathrm{~ms}$ pulse width, $300 \mathrm{~Hz}$ ). For the two lowest pulse amplitudes, the number of trials was set to match the total charge transfer achieved in 50 trials of $60 \mu \mathrm{A}$ pulses (i.e., 150 trials for $20 \mu \mathrm{A}$ pulses; 75 trials for $40 \mu \mathrm{A}$ pulses). The main patch (yellow star) and distant patches (arrowheads) were consistent in location for the three current amplitudes (Fig. 9D). Nevertheless, activation maps increased in size with current amplitude (Fig. $9 E)$. These size differences were most apparent for distant patches, particularly at $4 \mathrm{~mm}$ from the stimulation site (Fig. $9 F$ ).

\section{Train duration}

Four train durations $(18,38,75,150$ pulses/train) were tested while other ICMS parameters were constant $(1000 \mu \mathrm{m}$ depth, 40 $\mu \mathrm{A}, 0.2 \mathrm{~ms}$ pulse width, $300 \mathrm{~Hz}$ ). For the three shortest trains, the number of trials was set to match the total charge transfer achieved in 50 trials of 150 pulses/train (i.e., 417 trials for 18 

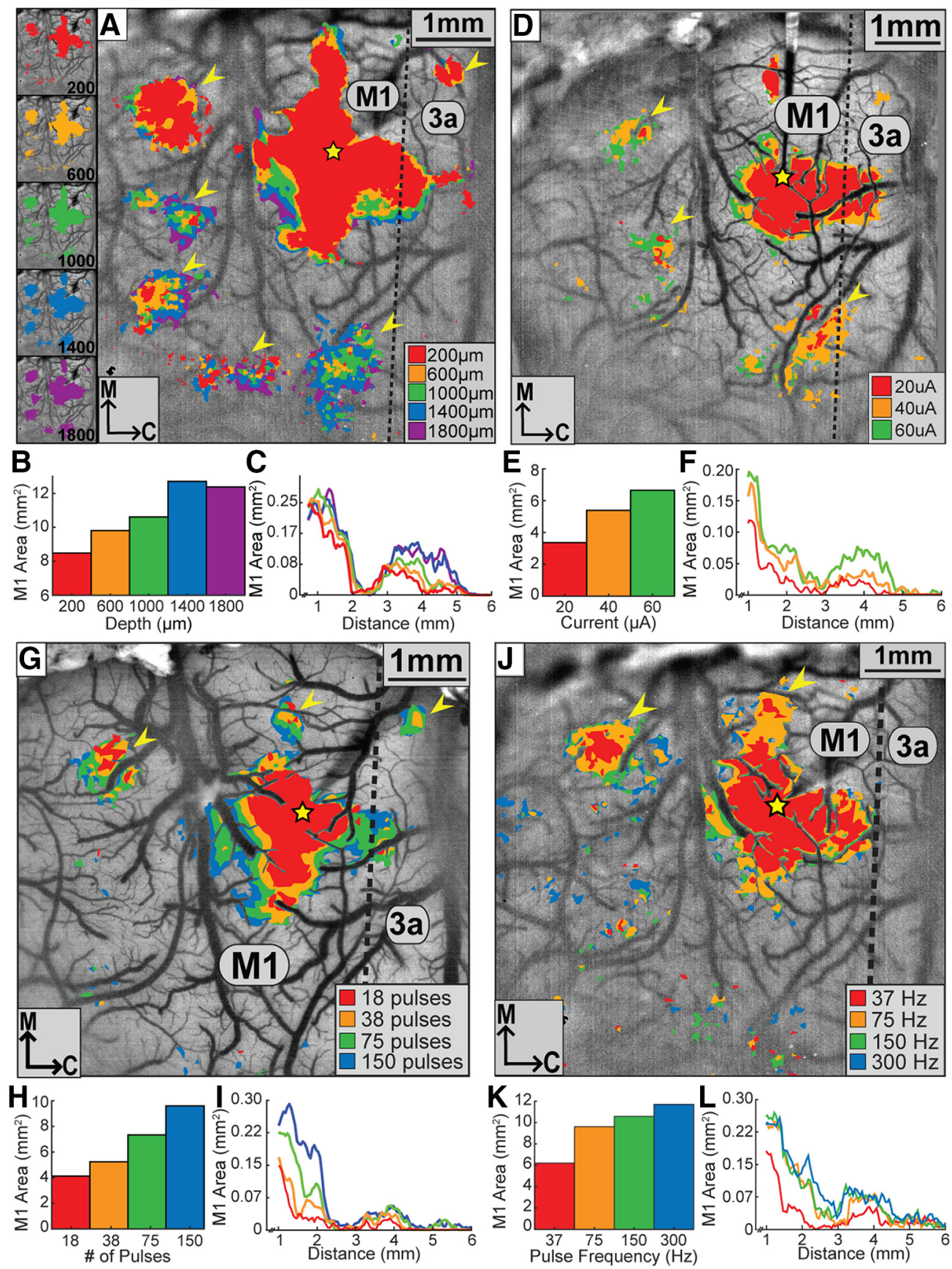

Figure 9. Activation maps are conserved across stimulation parameters. $\boldsymbol{A}-\boldsymbol{C}$, Microelectrode depth. $\boldsymbol{A}$, left column, Activation maps evoked in response to stimulation at five cortical depths. Depth $(\mu \mathrm{m})$ from surface of cortex is in the bottom right corner of each activation map. The five activation maps are superimposed onto each other in the main panel. Yellow arrowheads point to distant patches of activation. $\boldsymbol{B}$, Size of activation maps increased with microelectrode depth (up to $1400 \mu \mathrm{m}$ ). $\boldsymbol{C}$, Line plot colors match bar colors in $\boldsymbol{B}$. Activation maps had a spatial range that extended up to $5 \mathrm{~mm}$ from the stimulation site. The two deepest stimulation locations activated the most amount of cortex, which was most evident 3-5 mm from the stimulation site. $\mathbf{D}-\boldsymbol{F}$, Current amplitude. $\boldsymbol{D}$, Activation maps evoked in response to the same ICMS train at 3 current amplitudes: 20,40, and $60 \mu \mathrm{A}$. Number of trials was set to match the charge delivered in each condition ( 50 trials of $60 \mu \mathrm{A}, 75$ trials of $40 \mu \mathrm{A}$, and 150 trials of $20 \mu \mathrm{A}$ ). $\boldsymbol{E}$, Size of activation maps increased with pulse amplitude. $\boldsymbol{F}$, Line plot colors match bar colors in $\boldsymbol{E}$. Activation maps had comparable spatial ranges, but the $60 \mu \mathrm{A}$ condition activated larger distant patches (3-5 mm interval). The activation map of the $20 \mu \mathrm{A}$ condition was relatively small throughout its spatial range. G-I, Train duration. G. Activation maps evoked in response to $M 1$ stimulation with varying train durations: $18,38,75$, and 150 pulses/train. The number of trials was set to match the charge delivered in each condition (50 trials of 150 pulses/train, 100 trials of 75 pulses/train, 200 trials of 38 pulses/train, and 400 trials of 18 pulses/train). $\boldsymbol{H}$, Size of activation maps increased with train duration. $\boldsymbol{I}$, Line plot colors match bar colors in $\boldsymbol{H}$. The longest train durations (75 and 150 pulses/ train) activated the largest main patches as evident from surface area differences at $1-2 \mathrm{~mm}$. $J-L$, Pulse frequency. $J$, Activation maps evoked in response to M1 stimulation with varying pulse frequencies: $37,75,150$, and $300 \mathrm{~Hz}$. To account for longer activation times associated with the lower pulse frequencies, activation maps include any pixels activated within $6 \mathrm{~s}$ of ICMS onset. $\boldsymbol{K}$, Size of activation maps increased with pulse frequency. $\boldsymbol{L}$, Line plot colors match bar colors in $\boldsymbol{K}$. Activation maps had comparable spatial ranges except for the $37 \mathrm{~Hz}$ condition, which was smaller throughout its range. Experiments in this figure were conducted in the right hemisphere of monkey D. Panels are reflected for consistency with other figures. Shadow of the stimulating microelectrode is evident in $\mathbf{D}, \mathbf{G}$. 
pulses; 198 trials for 38 pulses; 100 trials for 75 pulses). Spatial organization was conserved for the main patch (Fig. 9G, star) and the distant patches (Fig. 9G, arrowhead) across train durations. Nevertheless, activation maps increased in size consistently with train duration (Fig. 9H). The most apparent size difference was within 1-2 $\mathrm{mm}$ from the stimulation site and therefore within the main patch (Fig. 9I).

\section{Pulse frequency}

Four pulse frequencies $(37,75,150,300 \mathrm{~Hz})$ were tested while other stimulation parameters were constant $(1000 \mu \mathrm{m}$ depth, 150 biphasic pulses, $0.2 \mathrm{~ms}$ pulse width, $60 \mu \mathrm{A}$ ). Train durations were adjusted to achieve 150 pulses/trial. Number of trials was consistent between conditions. Spatial organization was conserved for the main patch (Fig. 9J, star) and for distant patches (Fig. 9J, arrowheads) across the pulse frequencies tested. Activation maps increased in size with pulse frequency (Fig. $9 \mathrm{~K}$ ). The increase in size of the activation map occurred at all spatial intervals from the stimulation site (Fig. 9L).

\section{Discussion}

We investigated the intrinsic connectivity of the M1 forelimb representation in an effort to understand organizational principles of intrinsic M1 networks. ICMS+ISOI allowed us to measure connectivity in vivo and at high spatial resolution. ICMS+ ISOI conferred scale (i.e., number of sites), field-of-view, and spatial resolution needed to identify connectivity rules. These rules converged onto an overarching principle where intrinsic M1 connectivity links functionally matching patches.

\section{Tracing connectivity with ICMS + ISOI}

ICMS+imaging remedies a critical bottleneck that arises in studying cortical connectivity with a limited number of distinguishable tracers. Although ICMS+imaging is a relatively new approach, its reliability has been shown in several cortical networks. For example, ICMS+fMRI has been successfully used in mapping the connectivity of frontal eye fields with visual areas (Ekstrom et al., 2008); the connectivity of primary visual cortex (V1) with extrastriate cortex (Tolias et al., 2005); the intrinsic connectivity of the face-processing and body-processing systems in inferotemporal cortex (Moeller et al., 2008; Premereur et al., 2016). Similarly, ICMS + ISOI has been used to reveal parietalfrontal connectivity (Stepniewska et al., 2011) and connectivity of somatosensory areas (Friedman et al., 2020). Although fMRI and ISOI are no match for the spatial resolution achieved with tracers, the activation patches revealed with ICMS+imaging correspond closely with patches monosynaptically labeled from tracer injections (Stepniewska et al., 2009; Négyessy et al., 2013; Grimaldi et al., 2016). ICMS+imaging is therefore a suitable alternative to tracers that is particularly desirable for investigating large numbers of sites with overlapping connections; precisely what was needed here for the M1 forelimb representation.

We favored ISOI over fMRI for two reasons. First, ISOI provides superior spatial resolution $(13 \mu \mathrm{m} /$ pixel here $)$. Second, ISOI detects hemodynamic responses to microstimulation current amplitudes $\geq 10 \times$ smaller than needed for a reliable BOLD response (Tolias et al., 2005; Matsui et al., 2011; Premereur et al., 2016). The connectivity patterns that we identified in 4 sites within the digit representations of areas $3 \mathrm{~b}$ and 1 corresponded closely with feedforward and feedback connections of single digit representations (Liao et al., 2013; Négyessy et al., 2013). Moreover, our activation maps showed little/no evidence of activation spreading trans-synaptically or by fibers of passage. Either of these issues would have resulted in activation patches in locations not supported by tracer injections (e.g., inappropriate digit

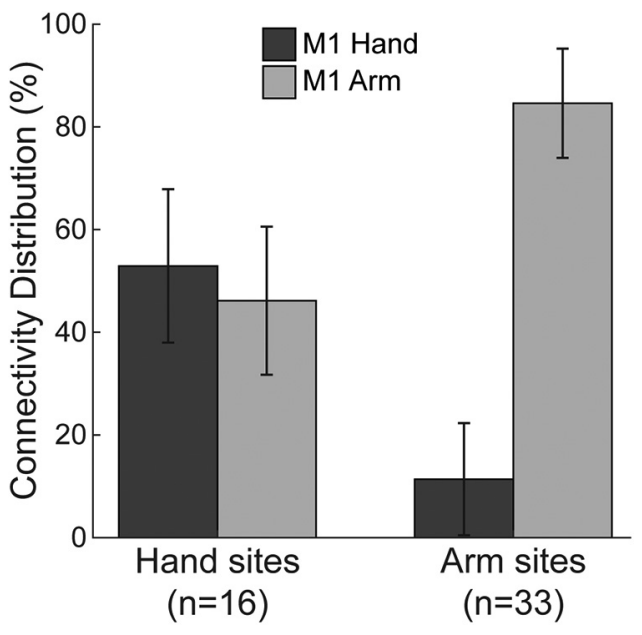

Figure 10. Summary of intrinsic M1 connectivity for arm and hand zones. Results (mean \pm 1 SD) are based on quantification of activation maps from all arm sites $(n=33)$ and hand site $(n=16)$. Arm sites mostly activated patches in the arm zone indicating preferential connectivity between functionally matching patches. Hand sites showed connectivity between functionally matching patches and non-matching patches.

representation). We also showed that systematic manipulation of microstimulation parameters mostly affected patch size (Fig. 9) without disturbing the spatial organization of activation maps. These observations support the likelihood that our activation maps were primarily driven by monosynaptic connections from the stimulation site. We note that the trans-synaptic effects reported in visual areas in response to lateral geniculate stimulation (Logothetis et al., 2010) may have resulted from relatively high current amplitudes or may reflect genuine differences in signal propagation from thalamic stimulation versus cortical stimulation. It is therefore important in future work to directly compare stimulation evoked activation and tracer injections in the same cortical sites. Perhaps of equal importance is the need to directly compare stimulation evoked activation with other causal methods such as optogenetics and with non-causal methods such as time-series correlations from resting state activity.

\section{Intrinsic M1 connectivity is patchy}

Stimulation of M1 forelimb sites activated patches within M1. Activation patterns were consistent with previous M1 tracer injections that labeled clusters of cell-bodies and axon-terminals, which appeared as patches in tangential sections or radial columns in cross sections (Huntley and Jones, 1991; Keller, 1993; Lund et al., 1993). The patchy pattern of intrinsic M1 connections has generally been underreported as M1 studies have largely focused on extrinsic connections (e.g., cortical-cortical, thalamocortical, corticospinal). A case-in-point is our own M1 injections that unmistakably labeled patches of cell bodies within M1 (Gharbawie et al., 2011, their Figs. 6, 7), but that pattern was scarcely discussed in favor of focusing on cortical inputs to M1.

We note that others have argued in favor of an alternative organization wherein a point within the M1 forelimb representation is connected widely with the rest of the forelimb representation (Capaday et al., 2009, 2011). This framework was grounded in the distribution of synaptic boutons throughout the lengths of projection axons (Capaday et al., 2009). Our results from dozens of stimulation sites in M1 indicate that activation patterns are patchy. This raises the possibility that ICMS evoked responses in locations where boutons clustered and/or had high synaptic strength. Indeed, close examination of the distribution of boutons in Capaday et al. (2009, their Figs. 3, 4, 8) shows that beyond 
the main tracer uptake zone $(\sim 1.5 \mathrm{~mm})$, zones of labeled synaptic boutons were punctuated by label-devoid zones. This pattern of labeling is generally regarded as patchy and likely reflects the spatial organization of the long-range horizontal connections.

The main activation patch that surrounded our ICMS site extended tangentially for $\sim 1.0 \mathrm{~mm}$ radius. The size of the main patch was consistent with the signal propagation patterns recorded with microelectrode arrays in response to focal stimulation in M1 (Capaday et al., 2011; Hao et al., 2016). The main patch is perhaps equivalent to the "halo" of dense labeling that results from tracer injections. Consistent organization of the main patch across dozens of M1 sites indicates that any point within M1 is endowed with short-range horizontal connections that are dense and have a high concentration of synaptic boutons (Gatter et al., 1978; Capaday et al., 2009). It is important to note that the main patch extended well-beyond the estimated range of passive current spread (150-500 $\mu \mathrm{m})$ for our current amplitudes (Stoney et al., 1968). Given the point spread function of intrinsic signals (Grinvald et al., 1994; Johnson and Frostig, 2016), it is possible that the main patch overestimated the range of the horizontal connections that surround each stimulation site but perhaps by no more than $175 \mu \mathrm{m}$ (Vazquez et al., 2014).

Beyond the main patch, intrinsic M1 connectivity was quite distinctive for arm sites and hand sites. Stimulation in arm sites typically drove multiple distant patches that were in some instances as far as $7 \mathrm{~mm}$ from the stimulation site. In contrast, hand sites scarcely activated patches $3 \mathrm{~mm}$ beyond the stimulation sites. In either case, the long-range projections of pyramidal neurons were likely the substrate for transmission of activity from the ICMS site to distant patches (Keller and Asanuma, 1993; Hao et al., 2016).

\section{Intrinsic M1 connectivity is functionally organized}

Intrinsic M1 connectivity is closely tied to the somatotopic organization of the forelimb representation. We observed from dozens of arm sites that activation patches coincided primarily with M1 arm zones. Given that arm sites have the largest footprint within the forelimb representation, we posit that preferential connectivity between functionally matching patches is a prominent organizational principle in M1 (Fig. 10). This point is particularly evident if we consider that the distant patches of arm sites were overwhelmingly located in M1 arm zones. These patches seemed to avoid M1 hand zones even if they were closer to the stimulation site than arm zones. Nevertheless, the connectivity portfolio of hand sites included a larger proportion of functionally non-matching patches as compared with the connectivity portfolio of arm sites. These differences likely reflect functional specializations of the arm and the hand. Connectivity between non-matching zones has been reported for the digit representation in macaque monkeys, and the wrist and arm representations in cats (Huntley and Jones, 1991; Keller, 1993; Capaday et al., 2009). However, these studies did not provide the quantification needed for determining the proportion of connectivity between matching patches and between non-matching patches. Based on the quantitative results in our study, we propose that intrinsic networks of the M1 forelimb representation are spatially organized to serve interactions between functionally matching patches and to a lesser extent serve interactions between functionally non-matching patches.

The connectivity of arm and hand sites differed in spatial range and in spatial organization. This result is intuitive if we consider (1) the nested somatotopy that we reported for arm and hand zones, and (2) the preferential connectivity between functionally matching patches. Had we found that stimulation sites drove spatially-shifted copies of the same activation map, then there would have been comparable likelihood of connectivity between functionally non-matching patches and between functionally matching patches. However, our activation maps do not support such organization. Moreover, the four parallel corticocortical networks previously described for the M1 hand representation (Dea et al., 2016; Hamadjida et al., 2016) are commensurate with the existence of multiple intrinsic connectivity patterns in M1. An important next step is to determine relationships between intrinsic M1 networks and the networks that M1 forms with other cortical areas. The advantages of the ICMS+ISOI connectivity mapping paradigm are bound to be valuable in such investigations.

\section{Conclusions}

The intrinsic networks of the M1 forelimb representation appear to be governed by a general principle: connectivity is patchy and primarily links functionally matching patches. Thus, the intrinsic connectivity of M1 is intimately linked to the somatotopic organization of the M1 forelimb representation. The relationship between connectivity and other defining features such as functional architecture is a well-established organizational principle in sensory cortex. For example, intrinsic connections of V1 preferentially link matching columns as defined with cytoarchitecture (e.g., cytochrome oxidase blobs) or with neurophysiology (e.g., orientation domains; Livingstone and Hubel, 1984; Gilbert and Wiesel, 1989; Malach et al., 1993; Bosking et al., 1997). The spatial periodicity of the connections in relation to the cytoarchitectonic and functional maps is considered part-in-parcel of the modular organization of sensory cortex. Accordingly, some of the fingerprints of modularity are inherent to the intrinsic M1 organization described here.

\section{References}

Aroniadou VA, Keller A (1993) The patterns and synaptic properties of horizontal intracortical connections in the rat motor cortex. J Neurophysiol 70:1553-1569.

Baldwin MKL, Cooke DF, Krubitzer L (2017) Intracortical microstimulation maps of motor, somatosensory, and posterior parietal cortex in tree shrews (Tupaia belangeri) reveal complex movement representations. Cereb Cortex 27:1439-1456.

Bosking WH, Zhang Y, Schofield B, Fitzpatrick D (1997) Orientation selectivity and the arrangement of horizontal connections in tree shrew striate cortex. J Neurosci 17:2112-2127.

Brock AA, Friedman RM, Fan RH, Roe AW (2013) Optical imaging of cortical networks via intracortical microstimulation. J Neurophysiol 110: 2670-2678.

Capaday C, Devanne H, Bertrand L, Lavoie BA (1998) Intracortical connections between motor cortical zones controlling antagonistic muscles in the cat: a combined anatomical and physiological study. Exp Brain Res 120:223-232.

Capaday C, Ethier C, Brizzi L, Sik A, van Vreeswijk C, Gingras D (2009) On the nature of the intrinsic connectivity of the cat motor cortex: evidence for a recurrent neural network topology. J Neurophysiol 102:2131-2141.

Capaday C, van Vreeswijk C, Ethier C, Ferkinghoff-Borg J, Weber D (2011) Neural mechanism of activity spread in the cat motor cortex and its relation to the intrinsic connectivity. J Physiol 589:2515-2528.

Dancause N, Duric V, Barbay S, Frost SB, Stylianou A, Nudo RJ (2008) An additional motor-related field in the lateral frontal cortex of squirrel monkeys. Cereb Cortex 18:2719-2728.

Dea M, Hamadjida A, Elgbeili G, Quessy S, Dancause N (2016) Different patterns of cortical inputs to subregions of the primary motor cortex hand representation in Cebus apella. Cereb Cortex 26:1747-1761.

Donoghue JP, Leibovic S, Sanes JN (1992) Organization of the forelimb area in squirrel monkey motor cortex: representation of digit, wrist, and elbow muscles. Exp Brain Res 89:1-19. 
Douglas RJ, Martin KAC (2004) Neuronal circuits of the neocortex. Annu Rev Neurosci 27:419-451.

Dum RP, Strick PL (2005) Frontal lobe inputs to the digit representations of the motor areas on the lateral surface of the hemisphere. J Neurosci 25:1375-1386.

Ekstrom LB, Roelfsema PR, Arsenault JT, Bonmassar G, Vanduffel W (2008) Bottom-up dependent gating of frontal signals in early visual cortex. Science 321:414-417.

Friedman RM, Morone KA, Gharbawie OA, Roe AW (2020) Mapping mesoscale cortical connectivity in monkey sensorimotor cortex with optical imaging and microstimulation. J Comp Neurol. Advance online publication. Retrieved April 7, 2020. doi: 10.1002/cne.24918.

Gatter KC, Sloper JJ, Powell TP (1978) The intrinsic connections of the cortex of area 4 of the monkey. Brain 101:513-541.

Gharbawie OA, Stepniewska I, Qi H, Kaas JH (2011) Multiple parietal-frontal pathways mediate grasping in macaque monkeys. J Neurosci 31:1166011677.

Gilbert CD, Wiesel TN (1989) Columnar specificity of intrinsic horizontal and corticocortical connections in cat visual cortex. J Neurosci 9:2432-2442.

Gould HJ, Cusick CG, Pons TP, Kaas JH (1986) The relationship of corpus callosum connections to electrical stimulation maps of motor, supplementary motor, and the frontal eye fields in owl monkeys. J Comp Neurol 247:297-325.

Graziano MSA, Taylor CSR, Moore T (2002) Complex movements evoked by microstimulation of precentral cortex. Neuron 34:841-851.

Grimaldi P, Saleem KS, Tsao D (2016) Anatomical connections of the functionally defined "face patches" in the macaque monkey. Neuron 90:13251342.

Grinvald A, Lieke EE, Frostig RD, Hildesheim R (1994) Cortical point-spread function and long-range lateral interactions revealed by real-time optical imaging of macaque monkey primary visual cortex. J Neurosci 14:25452568.

Hamadjida A, Dea M, Deffeyes J, Quessy S, Dancause N (2016) Parallel cortical networks formed by modular organization of primary motor cortex outputs. Curr Biol 26:1737-1743.

Hao Y, Riehle A, Brochier TG (2016) Mapping horizontal spread of activity in monkey motor cortex using single pulse microstimulation. Front Neural Circuits 10:104.

He SQ, Dum RP, Strick PL (1995) Topographic organization of corticospinal projections from the frontal lobe: motor areas on the medial surface of the hemisphere. J Neurosci 15:3284-3306.

Huntley GW, Jones EG (1991) Relationship of intrinsic connections to forelimb movement representations in monkey motor cortex: a correlative anatomic and physiological study. J Neurophysiol 66:390-413.

Johnson BA, Frostig RD (2016) Long, intrinsic horizontal axons radiating through and beyond rat barrel cortex have spatial distributions similar to horizontal spreads of activity evoked by whisker stimulation. Brain Struct Funct 221:3617-3639.

Kaas JH, Nelson RJ, Sur M, Lin CS, Merzenich MM (1979) Multiple representations of the body within the primary somatosensory cortex of primates. Science 204:521-523.

Keller A (1993) Intrinsic connections between representation zones in the cat motor cortex. Neuroreport 4:515-518.

Keller A, Asanuma H (1993) Synaptic relationships involving local axon collaterals of pyramidal neurons in the cat motor cortex. J Comp Neurol 336:229-242.

Kwan HC, MacKay WA, Murphy JT, Wong YC (1978) Spatial organization of precentral cortex in awake primates. II. Motor outputs. J Neurophysiol 41:1120-1131.

Liao CC, Gharbawie OA, Qi H, Kaas JH (2013) Cortical connections to single digit representations in area $3 \mathrm{~b}$ of somatosensory cortex in squirrel monkeys and prosimian galagos. J Comp Neurol 521:3768-3790.

Livingstone MS, Hubel DH (1984) Specificity of intrinsic connections in primate primary visual cortex. J Neurosci 4:2830-2835.

Logothetis NK, Augath M, Murayama Y, Rauch A, Sultan F, Goense J, Oeltermann A, Merkle H (2010) The effects of electrical microstimulation on cortical signal propagation. Nat Neurosci 13:1283-1291.
Lund JS, Yoshioka T, Lund JS, Yoshioka T, Levitt JB, Lund JS, Levitt JB (1993) Comparison of intrinsic connectivity in different areas of macaque monkey cerebral cortex. Cereb Cortex 3:148-162.

Malach R, Amir Y, Harel M, Grinvald A (1993) Relationship between intrinsic connections and functional architecture revealed by optical imaging and in vivo targeted biocytin injections in primate striate cortex. Proc Natl Acad Sci USA 90:10469-10473.

Matsui T, Tamura K, Koyano KW, Takeuchi D, Adachi Y, Osada T, Miyashita Y (2011) Direct comparison of spontaneous functional connectivity and effective connectivity measured by intracortical microstimulation: an fMRI study in macaque monkeys. Cereb Cortex 21:2348-2356.

Merzenich MM, Kaas JH, Sur M, Lin CS (1978) Double representation of the body surface within cytoarchitectonic area $3 \mathrm{~b}$ and 1 in "SI" in the owl monkey (Aotus trivirgatus). J Comp Neurol 181:41-73.

Moeller S, Freiwald WA, Tsao DY (2008) Patches with links: a unified system for processing faces in the macaque temporal lobe. Science 320:1355-1359.

Négyessy L, Pálfi E, Ashaber M, Palmer C, Jákli B, Friedman RM, Chen LM, Roe AW (2013) Intrinsic horizontal connections process global tactile features in the primary somatosensory cortex: neuroanatomical evidence. J Comp Neurol 521:2798-2817.

Nudo RJ, Milliken GW (1996) Reorganization of movement representations in primary motor cortex following focal ischemic infarcts in adult squirrel monkeys. J Neurophysiol 75:2144-2149.

Nudo RJ, Milliken GW, Jenkins WM, Merzenich MM (1996) Use-dependent alterations of movement representations in primary motor cortex of adult squirrel monkeys. J Neurosci 16:785-807.

Park MC, Belhaj-Saïf A, Gordon M, Cheney PD (2001) Consistent features in the forelimb representation of primary motor cortex in rhesus macaques. J Neurosci 21:2784-2792.

Premereur E, Taubert J, Janssen P, Vogels R, Vanduffel W (2016) Effective connectivity reveals largely independent parallel networks of face and body patches. Curr Biol 26:3269-3279.

Sakas DE, Borges LF, Zervas NT (1990) Biologically inert synthetic dural substitutes. Appraisal of a medical-grade aliphatic polyurethane and a polysiloxane-carbonate block copolymer. J Neurosurg 73:936-941.

Sessle BJ, Wiesendanger M (1982) Structural and functional definition of the motor cortex in the monkey (Macaca fascicularis). J Physiol 323:245-265.

Stepniewska I, Preuss TM, Kaas JH (1993) Architectonics, somatotopic organization, and ipsilateral cortical connections of the primary motor area (M1) of owl monkeys. J Comp Neurol 330:238-271.

Stepniewska I, Fang PC, Kaas JH (2005) Microstimulation reveals specialized subregions for different complex movements in posterior parietal cortex of prosimian galagos. Proc Natl Acad Sci USA 102:4878-4883.

Stepniewska I, Cerkevich CM, Fang PCY, Kaas JH (2009) Organization of the posterior parietal cortex in galagos: II. Ipsilateral cortical connections of physiologically identified zones within anterior sensorimotor region. J Comp Neurol 517:783-807.

Stepniewska I, Friedman RM, Gharbawie OA, Cerkevich CM, Roe AW, Kaas JH (2011) Optical imaging in galagos reveals parietal-frontal circuits underlying motor behavior. Proc Natl Acad Sci USA 108:E5725-E5732.

Stoney SD, Thompson WD, Asanuma H (1968) Excitation of pyramidal tract cells by intracortical microstimulation: effective extent of stimulating current. J Neurophysiol 31:659-669.

Strick PL, Preston JB (1982) Two representations of the hand in area 4 of a primate. I. Motor output organization. J Neurophysiol 48:139-149.

Sur M, Nelson RJ, Kaas JH (1982) Representations of the body surface in cortical areas $3 \mathrm{~b}$ and 1 of squirrel monkeys: comparisons with other primates. J Comp Neurol 211:177-192.

Tolias AS, Sultan F, Augath M, Oeltermann A, Tehovnik EJ, Schiller PH, Logothetis NK (2005) Mapping cortical activity elicited with electrical microstimulation using fMRI in the macaque. Neuron 48:901-911.

Vazquez AL, Fukuda M, Crowley JC, Kim SG (2014) Neural and hemodynamic responses elicited by forelimb- and photo-stimulation in channelrhodopsin-2 mice: Insights into the hemodynamic point spread function. Cereb Cortex 24:2908-2919.

Weiss DS, Keller A (1994) Specific patterns of intrinsic connections between representation zones in the rat motor cortex. Cereb cortex 4:205-214. 